\title{
Coordinated Model Predictive Control of Aircraft Gas Turbine Engine and Power System
}

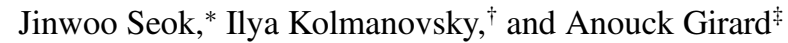 \\ University of Michigan, Ann Arbor, Michigan 48109-2140
}

DOI: $\underline{10.2514 / 1 . G 002562}$

\begin{abstract}
Motivated by the growing need to accommodate large transient thrust and electrical load requests in future more-electric aircraft, a coordinated control strategy for a gas turbine engine, generators, and energy storage is developed. An advanced two-generator configuration, with each generator connected to a shaft of the gas turbine engine, is treated. Model predictive control maximizes system performance and protects this system against constraint violations. The controller design is exploits rate-based linear prediction models. In addition, an auxiliary offset state improves the match between the linear prediction model and the nonlinear system. The auxiliary offset state allows the system to be controlled over the large operating range without requiring multiple linearizations/ controllers. The advantages of different energy storages are also compared to complement the two-generator configuration in a more electric aircraft. Primary results indicate that the coordinated model predictive control with an auxiliary offset state yields better performance than other control strategies, and it successfully controls the considered system to satisfy specified requirements over a large operating range. The battery-ultracapacitor pack allows improvement of the overall system performance.
\end{abstract}

\section{Introduction}

$\mathbf{I}_{\mathrm{a}}^{\mathrm{s}}$ $\mathrm{N}$ THE past few decades, the electrical power requirements for aircraft have been steadily increasing, and this growth has been concomitant with trends toward more-electric aircraft (MEA) and allelectric aircraft (AEA) [1]. A typical aircraft power system involves one or more generators connected to one or more gas turbine engines, which are integrated with energy storage elements that provide supplemental electrical power, a distribution system, and loads. The large electrical loads, including both steady and transient loads, affect the operation of the generators and of the gas turbine engines. For instance, large electrical load changes induce large torque disturbances on the gas turbine engine and can affect engine thrust and shaft speeds. These changes, in turn, affect the generators; hence, the system exhibits strong static and dynamic interactions. Thus, in the presence of large electrical loads, the interactions between the electrical system and the gas turbine engine have to be addressed for the efficient and safe operation of aircraft.

Our objective is to establish an integrated model-based control capability for an aircraft's propulsion and electrical power systems (including thrust generation, electric power generation, and energy storage) that improves the capability of the system to accommodate large transients, including those caused by large transient electrical loads, while maintaining the operation of the components and the overall system within a specified safe range by enforcing appropriately defined state and control constraints.

Specifically, this paper considers the development of an integrated control system that accommodates large steady and transient electrical loads; maintains aircraft flight performance by delivering requested thrust; enforces gas turbine engine constraints (e.g., surge margins), as well as electrical system constraints (e.g., component power limits); and reduces fuel consumption. To facilitate the achievement of these goals, an advanced two-shaft distributed

Received 21 October 2016; revision received 7 March 2017; accepted for publication 14 March 2017; published online 19 May 2017. Copyright (C) 2017 by the American Institute of Aeronautics and Astronautics, Inc. All rights reserved. All requests for copying and permission to reprint should be submitted to CCC at www.copyright.com; employ the ISSN 0731-5090 (print) or 1533-3884 (online) to initiate your request. See also AIAA Rights and Permissions www.aiaa.org/randp.

*Ph.D. Candidate, Department of Aerospace Engineering; sjinu@ umich. edu.

†rofessor, Department of Aerospace Engineering; ilya@umich.edu. Member AIAA.

${ }^{\ddagger}$ Associate Professor, Department of Aerospace Engineering; anouck@ umich.edu. Associate Fellow AIAA. generator configuration is considered, where one generator is connected to the high-pressure shaft (HPS) and the other is connected to the low-pressure shaft (LPS) of the gas turbine engine. This configuration affords an extra degree of freedom to accommodate the effects of large loads compared to the single-shaft configuration. Furthermore, it potentially achieves better fuel efficiency than the single-shaft configuration. In addition, the integration of highperformance storage elements that can react quickly to transient loads to assist the generators and the gas turbine engines is considered.

To control such an advanced system with two generators, a gas turbine engine, and energy storage, we define a power split strategy between the two generators based on the offline minimization of the fuel consumption and a rule-based strategy to determine when to charge and discharge the energy storage. To protect the engine and the electrical system components against constraint violation, a ratebased model predictive control (MPC) framework is exploited and several MPC controller designs are developed, validated on a nonlinear model of the system, and compared with each other. The proposed framework is flexible and modular, and it can accommodate other constraints not explicitly treated in the paper, such as temperature constraints in the engine or voltage stability constraints in the electrical system, provided the prediction model is updated with representations for these constraints. Because only linear MPC design techniques are employed, the controller implementation is feasible with standard quadratic programming solvers, which are a mature and reliable technology.

The system configuration of interest in this paper is illustrated in Fig. 1. The system consists of a single gas turbine engine, energy storage element(s), and two generators: one of which is attached to the LPS of the gas turbine engine, whereas the other is attached to the HPS of the gas turbine engine.

The growing electrical power requirements of MEA and AEA are highlighted in $[1,2]$. For instance, at least $1.6 \mathrm{MW}$ will be required for a next-generation 300-passenger aircraft [2]. Large electrical power is required for turboelectric propulsion. Three megawatt generators were considered in [3], and a 40.2 MW generator was planned in [4,5]. Electrical weapons systems for military applications also require large electrical power: from 0.025 to $4.5 \mathrm{MW}$, depending on the type [6]. Directed energy systems are one of the key 12 potential capability areas for the U.S. Air Force [7]. To deal with these large electrical loads on aircraft, integrated control of the aircraft's gas turbine engine, electrical power system, and thermal management is necessary. Challenges in aircraft engine control and integrated power and thermal management were discussed in [ $\underline{8}-11]$.

MPC-based approaches have been considered to develop solutions to many recent control problems, including gas turbine engine 


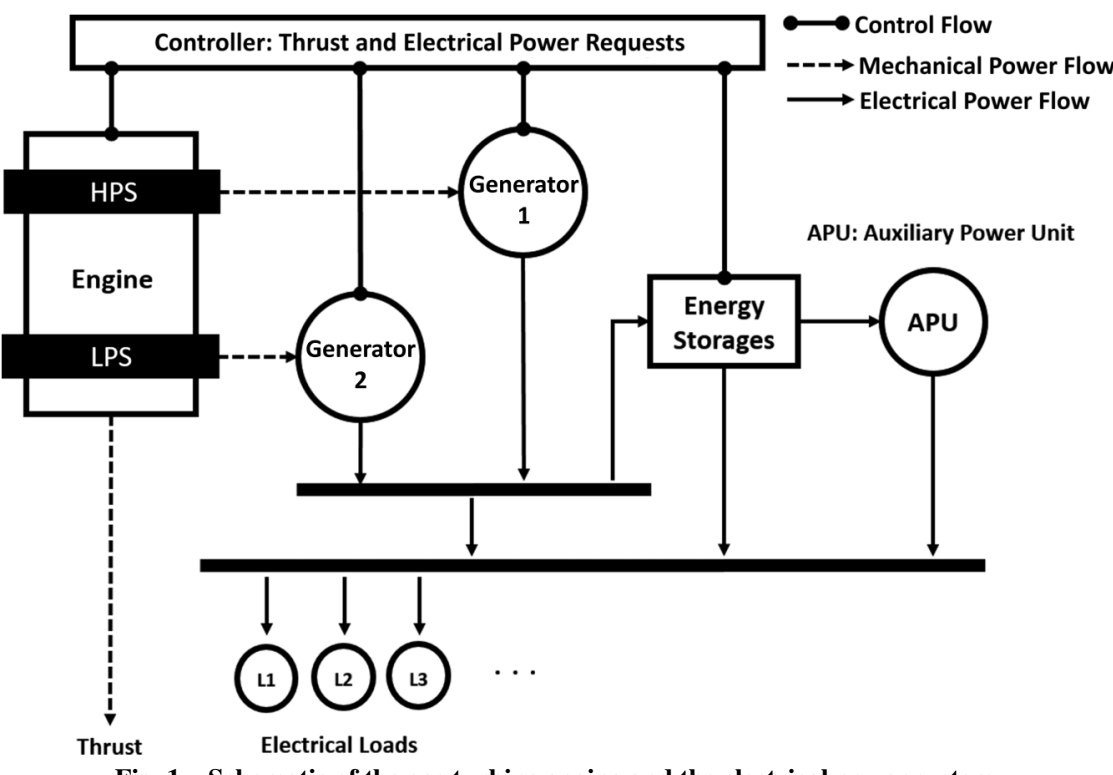

Fig. 1 Schematic of the gas turbine engine and the electrical power system.

control; see, e.g., [12-14]. Rate-based MPC allows setpoint tracking and was applied to turbofan engine clearance control in [13] and to turbocharged compression ignition engine control in [1] $]$. In this paper, the Multi-Parametric Toolbox (MPT) [16] is employed for computational implementation of a rate-based $\overline{\mathrm{MPC}}$ controller.

The two-generator configuration for aircraft, with one generator connected to the HPS and the other generator connected to the LPS of a gas turbine engine, was introduced in [17]. The challenges and possible research directions for unmanned aerial vehicles and MEA with a gas turbine engine, a two-generator configuration, and a battery (and/or supercapacitor) were discussed in [18]. The authors of [18] indicated the necessity of integrated control of the electrical system and the gas turbine engine due to interactions between both systems. In [19], the authors designed a voltage and current controller for the generators, and this work was extended in [20] to include a battery. The controller proposed in these references was based on a master-slave configuration for high-load situations. Existing publications on two-generator configurations focused primarily on the electrical system, especially voltage and current stability, and a control design exploiting batteries.

Integrated control of a gas turbine engine and electrical power system has been considered in some publications. The nonlinear MPC approach for a 166 MW heavy-duty single-shaft gas turbine powerplant based on simplified gas turbine engine and generator models was presented in [21]. The control goal was to supply allelectrical loads while maintaining the rotor speed, exhaust gas temperature, and turbine firing temperature by controlling airflow and fuel flow despite transient load changes. The control of the gas turbine engine and electrical system, which focused on their thermal management, for the U.S. Navy's future all-electric ship was considered in [22]. The importance of interactions between the gas turbine engine and the electrical system for aircraft was highlighted in [23], where the engine response when a step change reduction of electrical power occurred was simulated. In [24], an energy storage element (supercapacitor) was used to reduce the effects of dynamic loads on the engine using a proportional-integral (PI) supercapacitor controller. A load management system, which consisted of generators, contactors, buses, loads, and a battery for an aircraft electric power system was presented in [25]. The paper [25] focused on the electrical system of the aircraft, mainly controlling contactors for safety and reliability, using load shedding. The aircraft gas turbine engine modeling and control were discussed in [26].

In this paper, the following problem formulation is considered: Given a gas turbine engine; energy storage elements; two generators, with one connected to each shaft of the gas turbine engine; a requested thrust level; and (large) expected/requested electrical loads, determine the fuel-to-air ratio of the gas turbine engine, the input/output power of the energy storage elements, and the electrical power output of each generator to supply all the required electrical loads, maintain the requested thrust level, and minimize fuel consumption, subject to surge margin limits and other constraints.

The Simulink-based Toolbox for the Modeling and Analysis of Thermodynamic Systems (T-MATS) [27-29] is used for the gas turbine engine modeling and is supplemented by an electrical power system model in Simulink. T-MATS allows one to model both steadystate and dynamic gas turbine engine operation.

The original contributions of this paper are now highlighted. Most of the existing studies on the two-generator configuration are limited only to the electrical system. In this paper, the effects on the gas turbine engine are considered, and it is shown that coordinated control solutions can be developed to increase capability and efficiency of the system. An advanced electrical power system configuration with two generators and an electrical storage element is also treated, for which control designs based on MPC are developed that accomplish simultaneous tracking of requested thrust and electrical power output commands while satisfying the imposed component protection constraints within the engine and the electrical system, as well as minimizing fuel consumption. These designs account for static and dynamic interactions between the gas turbine engine, generators, and energy storage. The paper illuminates the link between energy storage characteristics and control performance.

Existing aircraft power systems are typically optimized on a quasistatic individual component basis. Here, a novel control system architecture based on the combination of a rate-based linear quadratic/ MPC controller, a power split map between generators optimized for steady-state operation, and a supervisory logic to govern energy storage charging/discharging is defined. The benefits of constrained coordinated control include the ability to handle load pulses of higher frequency and a larger magnitude than possible with existing systems.

Unlike many of the previous publications, system operation over a large static and dynamic range is considered in this paper. The MPC controller designs based on single linear and multiple linear prediction models are compared where the linear prediction models are obtained by applying system identification techniques. As the paper shows, the mismatch between the linear prediction models and the actual nonlinear system can be successfully handled by auxiliary offset states; in particular, the surge margin constraints can be robustly enforced. A novel linear transformation approach to match states of different linear prediction models is also proposed. This approach avoids the need for designing observers for nonphysical states of the individual models. Furthermore, the paper demonstrates that successful control of the system can be accomplished by using a single rate-based linear prediction model with lower computational and implementational complexity as compared to the switched MPC 
approach. Based on a comparison of the closed-loop response with the one from the classical linear quadratic regulator (LQR) controller, the advantages of MPC are highlighted. We validate the design in nonlinear model simulations over the large engine operating range while responding to large transient thrust and electrical power load commands. As considered case studies demonstrate, the MPC control design framework is systematic and expandable to include additional components.

This work extends and goes much beyond our previous work that appeared in a conference paper [30]. In particular, in this paper, the system configuration with the energy storage elements is treated, the power split strategy for the tradeoff between the fuel consumption and the surge margin (rather than just fuel consumption) is optimized, and MPC controllers for the large operating range of the engine are developed and demonstrated to accommodate simultaneous transients in electrical load and thrust. The rate-based MPC designs based on a single and multiple linear models are compared, and offset states are introduced to compensate for the differences in response between the linear model and the nonlinear system. The linear transformation of the linear models is also introduced to match the states of the multiple linear models with the physical states.

The organization of this paper is as follows. Section II describes the system models and how linear models are constructed to predict system response. Section III addresses the control design. Section IV presents the simulation results on the full nonlinear model. Finally,

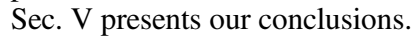

\section{Modeling}

In this section, models of the gas turbine engine, generators, and energy storage elements are described. A simple relationship between the shaft speeds of the gas turbine engine and the output power of the generators is used, assuming the dynamics of the generators are much faster than the dynamics of the gas turbine engine; and a first-order model is adopted to represent the dynamics of the energy storage elements. The engine model, generator models, and energy storage element models are assembled into a system level model in which one generator is connected to the HPS and the other generator is connected to the LPS of the gas turbine engine. Note that the assembled model is able to represent subsystem-level interactions visible in the simulation results.

Then, a linear model of the gas turbine engine with two generators is obtained via system identification, followed by a linear transformation of all the states of the linear model to physical states. Note that the identified linear model takes into account the interactions between the gas turbine engine and the generators. Finally, the identified linear model, the generator models, and the energy storage element models are combined to obtain the complete linear prediction model to be used in MPC control design.

\section{A. Gas Turbine Engine}

The JT9D gas turbine engine model provided with the T-MATS package [28] is used to represent engine dynamics. T-MATS is a Simulink-based tool for thermodynamic system simulation that was developed and released by NASA to facilitate research involving gas turbine engine simulations and control of the kind pursued in this paper. Unlike other packages, T-MATS is open to public use. It includes generic modeling libraries and is suitable for gas turbine engine modeling. The JT9D gas turbine engine model represents the dynamics of shaft speeds, pressures, and flows in various components of the engine and predicts engine thrust. The model is developed and verified based on data from the numerical propulsion system simulation [29]. The thrust $F_{g}$ is controlled using the fuel-to-air ratio (FAR) as a control input.

\section{B. Generators}

The two generators are each connected to different shafts of the gas turbine engine: one to the HPS, and one to the LPS. We refer to the generator that is connected to the HPS as the high-pressure shaft generator (HPSG) and the generator that is connected to the LPS as the low-pressure shaft generator (LPSG). Then, the power requested from the HPSG $P_{H \text { req }}$ and the power requested from the LPSG $P_{L \text { req }}$ are two additional control inputs in our system. The total output power from the generators $P_{G_{T}}$ is the sum of the output powers of the HPSG $P_{H}$ and LPSG $P_{L}$. The power difference between two generators $P_{D}$ is one of the outputs of the system and is defined as $P_{H}-P_{L}$.

Assuming that the dynamics of the generators are much faster than those of the gas turbine engine [31], a simple relationship between the shaft speeds of the gas turbine engine and the output power of the generators is adopted based on given efficiencies of the generators:

$$
\begin{aligned}
P_{H} & =N_{H} \times \tau_{E_{H}} \times \eta_{H}, \\
P_{L} & =N_{L} \times \tau_{E_{L}} \times \eta_{L}
\end{aligned}
$$

where $N_{H}, \tau_{E_{H}}$, and $\eta_{H}$ are, respectively, the shaft speed, the torque on the shaft, and the efficiency of the HPSG; and $N_{L}, \tau_{E_{L}}$, and $\eta_{L}$ are, respectively, the shaft speed, the torque on the shaft, and the efficiency of the LPSG. Thus, given electrical power outputs of the generators, the torques that the generators create on the gas turbine engine shafts can be computed according to

$$
\begin{aligned}
\tau_{E_{H}} & =\frac{P_{H}}{N_{H} \times \eta_{H}}, \\
\tau_{E_{L}} & =\frac{P_{L}}{N_{L} \times \eta_{L}}
\end{aligned}
$$

Note that the preceding electrical power system representation is suitable when given the specific control objectives in this paper, and it is justified by the timescale separation between the engine dynamics and the dynamics in the electrical power system. In the subsequent analysis and simulations, constant values of the efficiencies $\left(\eta_{H}=\eta_{L}=0.9\right)$ are assumed.

\section{Energy Storage Elements}

The energy storage element model is as follows:

$$
\frac{\mathrm{d} E_{j}}{\mathrm{~d} t}=-P_{j}
$$

where $E_{j}$ is the total energy stored in the energy storage $j, P_{j}$ is power to/from the energy storage $j$, and $j$ indicates the type of energy storage element. In this paper, a battery and/or ultracapacitor are exploited as the energy storage elements, so $j \in\{B, C\}$, where $B$ indicates the battery and $C$ indicates the ultracapacitor. Then, the state of charge (SOC) is given by

$$
\operatorname{SOC}_{j}=\frac{E_{j}}{E_{j_{\text {Max }}}}
$$

where $E_{j_{\text {Max }}}$ is the maximum energy that can be stored in the energy storage $j$. The total output/input power of the energy storage elements $P_{\mathrm{ES}_{T}}$ is the sum of the output/input power of all the energy storage elements. Then, the total output power $P_{T}$ is the sum of the total output powers from the generators $P_{G_{T}}$ and the total output/input powers of the energy storage elements $P_{\mathrm{ES}_{T}}$.

\section{Linear Design Model}

\section{System Identification and Linear Transformation}

The design of our MPC controller is based on a linear prediction model. Because our gas turbine engine model is essentially of the black-box type, either analytical or numerical (finite differencebased) linearization cannot be easily implemented. Consequently, the linear model is identified based on the input-output response data collected from the nonlinear model of the engine near a nominal operating point. The nominal operating point is the same as the one used for verifying the model in [29] $(27,593 \mathrm{lbf}$ thrust and FAR of 0.0187 ), and $P_{\text {Hreq }}=P_{\text {Lreq }}=0 \overline{\mathrm{MW}}$.

Our linear model to be identified has three inputs (FAR, $P_{H \text { req }}$, and $\left.P_{\text {Lreq }}\right)$ and five outputs [HPS speed, LPS speed, thrust, low-pressure 
compressor (LPC) surge margin, and high-pressure compressor (HPC) surge margin. The surge margins are added as outputs to the model to predict the evolution of the surge margin constraints over the prediction horizon.

To identify the linear prediction model at a given operating point, a system identification approach is followed. The input-output dataset is based on a $400 \mathrm{~s}$ trace generated when chirp signals are applied to each of FAR, $P_{H \text { req }}$, and $P_{L \text { req }}$ channels for $100 \mathrm{~s}$ individually; and then it is applied to all inputs in combination for another $100 \mathrm{~s}$. The magnitude of chirp signals is set to 0.001 for $\delta$ FAR and 0.5 for $\delta P_{H \text { req }}$ and $\delta P_{L \text { req }}$, where $\delta$ designates the deviation from steady-state values at the operating point. The chirp signal frequency ranges between 0 and $1.8 \mathrm{~Hz}$. After the set of input-output data is obtained by simulating the nonlinear model, mean removal is applied so that only variations from the steady state are reflected in the signals.

Based on such input-output data collected around a specific operating point, the linear model of order five is identified using the system identification toolbox in MATLAB, and it is verified to be both asymptotically stable and fully controllable. This identified linear model has the following form:

$$
\begin{aligned}
& \delta \dot{x}=A \delta x+B \delta u, \\
& \delta y=C \delta x
\end{aligned}
$$

where $\delta x$ is the state, $\delta u$ is the input deviations from the operating point, $\delta y$ is the output deviations from the operating point, $A \in \mathbb{R}^{5 \times 5}$, $B \in \mathbb{R}^{5 \times 3}$, and $C \in \mathbb{R}^{5 \times 5}$. The resulting linear model from system identification typically has $C \neq I$, which indicates that the states are not physical. Because models with physical states have advantages in terms of state estimation (e.g., nonphysical states must be estimated even if physical states are measured) and control design (e.g., switching between different linear state feedback controllers is straightforward), a state transformation is constructed to obtain $C=I$. Specifically, let $\delta z=\delta y$, so $\delta z$ is the physical state. Then,

$$
\delta z=C \delta x \Rightarrow C^{-1} \delta z=\delta x \Rightarrow \dot{C}^{-1} \delta z+C^{-1} \delta \dot{z}=\delta \dot{x}
$$

Substituting for $\delta \dot{x}$ from Eq. (ㅁ) yields

$$
\dot{C}^{-1} \delta z+C^{-1} \delta \dot{z}=A \delta x+B \delta u \Rightarrow C^{-1} \delta \dot{z}=A \delta x+B \delta u
$$

Because $\delta x=C^{-1} \delta z$,

$$
C^{-1} \delta \dot{z}=A C^{-1} \delta z+B \delta u \Rightarrow \delta \dot{z}=C A C^{-1} \delta z+C B \delta u
$$

Let $A^{\prime}=C A C^{-1}, B^{\prime}=C B$, and $\delta x=\delta z$. Then, the new system is as follows:

$$
\begin{aligned}
& \delta \dot{x}=A^{\prime} \delta x+B^{\prime} \delta u \\
& \delta y=C^{\prime} \delta x=I \delta x
\end{aligned}
$$

where now $\delta x$ is the physical state, and

$$
\delta x=\left[\begin{array}{c}
\delta x_{N_{H}} \\
\delta x_{N_{L}} \\
\delta x_{F g} \\
\delta x_{\mathrm{SM}} \\
\delta x_{\mathrm{SM}}
\end{array}\right], \quad \delta u=\left[\begin{array}{c}
\delta \mathrm{FAR} \\
\delta P_{H \text { req }} \\
\delta P_{L \text { req }}
\end{array}\right]
$$

Here $\delta x_{N_{H}}$ is the HPS speed deviation, $\delta x_{N_{L}}$ is the LPS speed deviation, $\delta x_{F g}$ is the thrust deviation, $\delta x_{\mathrm{SM}_{\mathrm{LPC}}}$ is the LPC surge margin deviation, and $\delta x_{\mathrm{SM}_{\mathrm{HPC}}}$ is the HPC surge margin deviation. The components of the control input vector are $\delta \mathrm{FAR}, \delta P_{H \text { req }}$, and $\delta P_{L \text { req }}$; and they represent the deviations in the respective inputs. Note that choosing the order of the linear model equal to five is essential for this transformation procedure to apply.

To confirm linear model accuracy, we have generated another $100 \mathrm{~s}$ trace of input-output data for validation purposes. This trace was constructed similarly to the one used to generate system identification data but with the chirp signals frequency range being between 0 and $3.2 \mathrm{~Hz}$, and chirp signals were applied to all inputs channels in combination for $100 \mathrm{~s}$. The agreement between the validation data and the identified linear model was $81.34 \%$ for HPS speed, $80.24 \%$ for LPS speed, $81.41 \%$ for thrust, $63.80 \%$ for LPC surge margin, and $82.62 \%$ for HPC surge margin. The agreement is defined in terms of the normalized root mean square error as

$$
\text { agreement }[\%]=100 \times\left(1-\frac{\|y-\hat{y}\|}{\left\|y-y_{\text {avg }}\right\|}\right)
$$

where $y$ is the measurement vector, $\hat{y}$ is the estimate vector, $y_{\text {avg }}$ is the mean of $y$, and $\|\cdot\|$ denotes the 2-norm applied to the respective vectors of measurements/estimates.

Figure 2 compares step responses of the linear model and T-MATS. These results were obtained at the operating point corresponding to FAR $=0.0187$, and $P_{H \text { req }}=P_{\text {Lreq }}=0$. The T-MATS initially runs at the steady state; then, step increments of the inputs $\delta \mathrm{FAR}=0.0001, \delta P_{H \text { req }}=0.1 \mathrm{MW}$, and $\delta P_{\text {Lreq }}=0.1 \mathrm{MW}$ are applied during the time period between 10 and $25 \mathrm{~s}$. The agreement between the nonlinear model (T-MATS) and the identified linear model is $94.75 \%$ for HPS speed, $86.74 \%$ for LPS speed, $93.37 \%$ for thrust, $74.15 \%$ for LPC surge margin, and $80.81 \%$ for HPC surge margin; and the average is $85.96 \%$. Note that, if the response of surge margins is not considered, the average agreement for the step responses between the nonlinear model and the identified linear model increases to $91.62 \%$, which is fairly accurate. A comparably larger mismatch of the surge margin response prediction is compensated by the auxiliary offset states (see Secs. III.D.2 and III.D.3). Furthermore, our controller is feedback-based, and feedback compensates for model inaccuracies.

To confirm model accuracy, we checked the sensitivity of the results to the choice of signals used for identification. Specifically, we considered 19 other random frequency subranges (within the overall 0-2.4 Hz range) for the chirp signal that was used to generate inputoutput data for identification. This did not substantially change the results against the validation data.

Steady-state values of thrust, LPC surge margin, and HPC surge margin deviations as functions of different $\delta \mathrm{FAR}, \delta P_{H}$, and $\delta P_{L}$ for different operating points based on the nonlinear model are shown in Fig. 3 . In the figure, different operating points (defined by different thrust levels) are indicated. As observed, the gas turbine engine with two generators is a highly nonlinear system. In particular, the static (dc) gains are different at different operating points defined by different thrust levels. Thus, multiple linear models may be needed to represent the response at different operating points.

\section{Combined Linear Model}

The linear model [Eq. (9)] is combined with the generator and energy storage elements models. The outputs of the integrated system are the thrust $F_{g}$, the total power $P_{T}$, the power difference between the two generators $P_{D}$, and the stored energy in energy storage elements $E_{j}$. The total power is $P_{H}+P_{L}+P_{j}=P_{H \text { req }}+P_{L \text { req }}+P_{\text {jreq }}$, and the power difference between the two generators is $P_{D}=P_{H}-P_{L}=P_{H \text { req }}-P_{L \text { req }}$. The combined model has the following form:

$$
\begin{aligned}
& {\left[\begin{array}{c}
\delta \dot{x} \\
\dot{E}_{j}
\end{array}\right]=\left[\begin{array}{cc}
A^{\prime} & 0 \\
0 & 0
\end{array}\right]\left[\begin{array}{c}
\delta x \\
E_{j}
\end{array}\right]+\left[\begin{array}{cc}
B^{\prime} & 0 \\
0 & -1
\end{array}\right]\left[\begin{array}{c}
\delta u \\
P_{\text {jreq }}
\end{array}\right],} \\
& {\left[\begin{array}{c}
\delta F_{g} \\
\delta P_{T} \\
\delta P_{D} \\
\delta P_{D} \\
E_{j}
\end{array}\right]=\left[\begin{array}{llllll}
0 & 0 & 1 & 0 & 0 & 0 \\
0 & 0 & 0 & 0 & 0 & 0 \\
0 & 0 & 0 & 0 & 0 & 0 \\
0 & 0 & 0 & 0 & 0 & 0 \\
0 & 0 & 0 & 0 & 0 & 1
\end{array}\right]\left[\begin{array}{c}
\delta x \\
E_{j}
\end{array}\right]+\left[\begin{array}{cccc}
0 & 0 & 0 & 0 \\
0 & 1 & 1 & 1 \\
0 & 1 & -1 & 0 \\
0 & 1 & -1 & 0 \\
0 & 0 & 0 & 0
\end{array}\right]\left[\begin{array}{c}
\delta u \\
P_{j \mathrm{req}}
\end{array}\right]}
\end{aligned}
$$

For control purposes, two outputs for the power difference between two generators $P_{D}$ are needed, as described in the next section. 

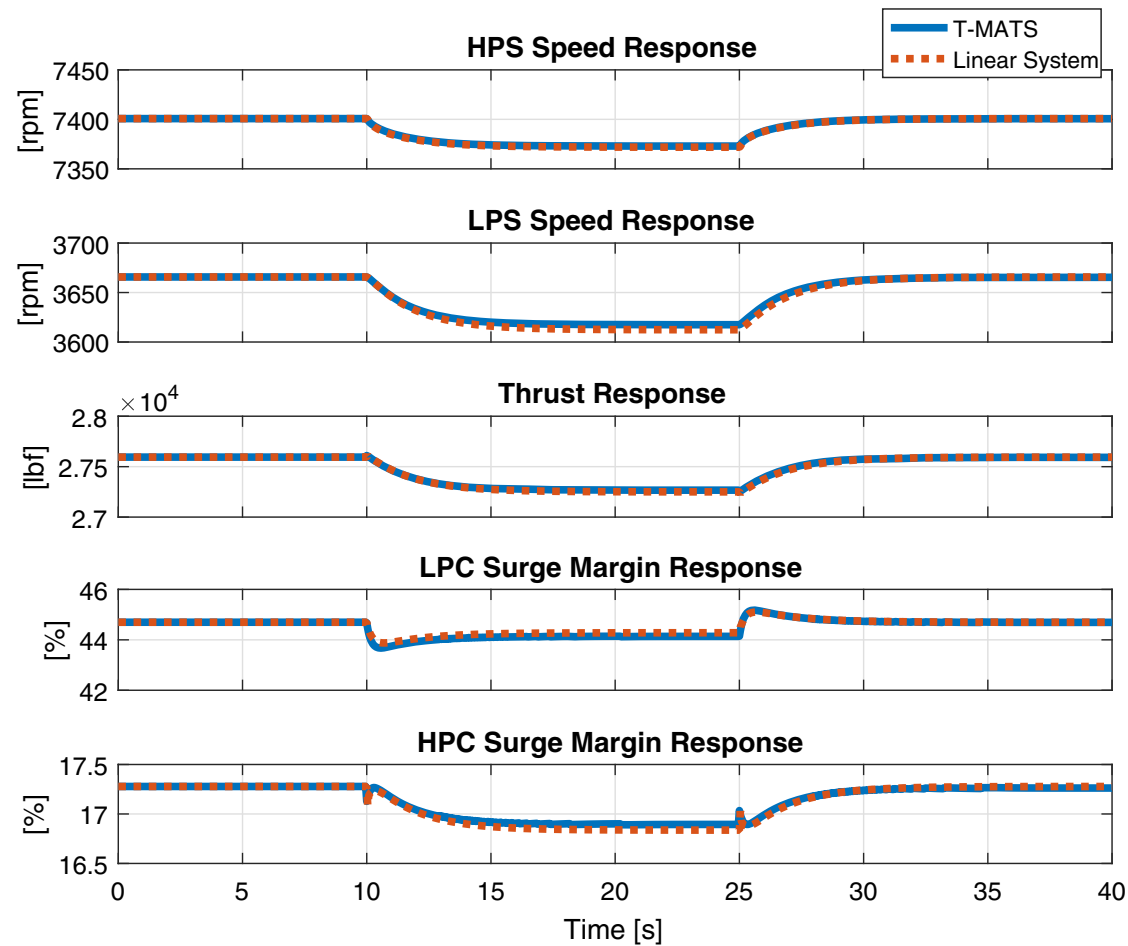

Fig. 2 Comparison of step responses of the linear and nonlinear models.
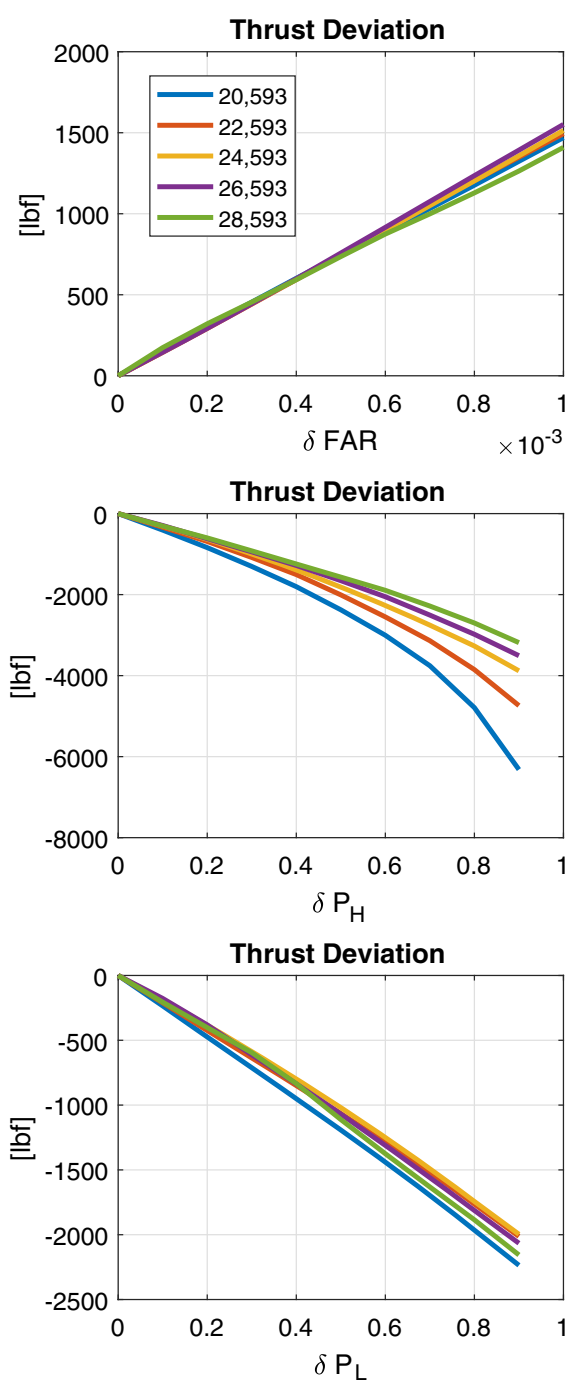
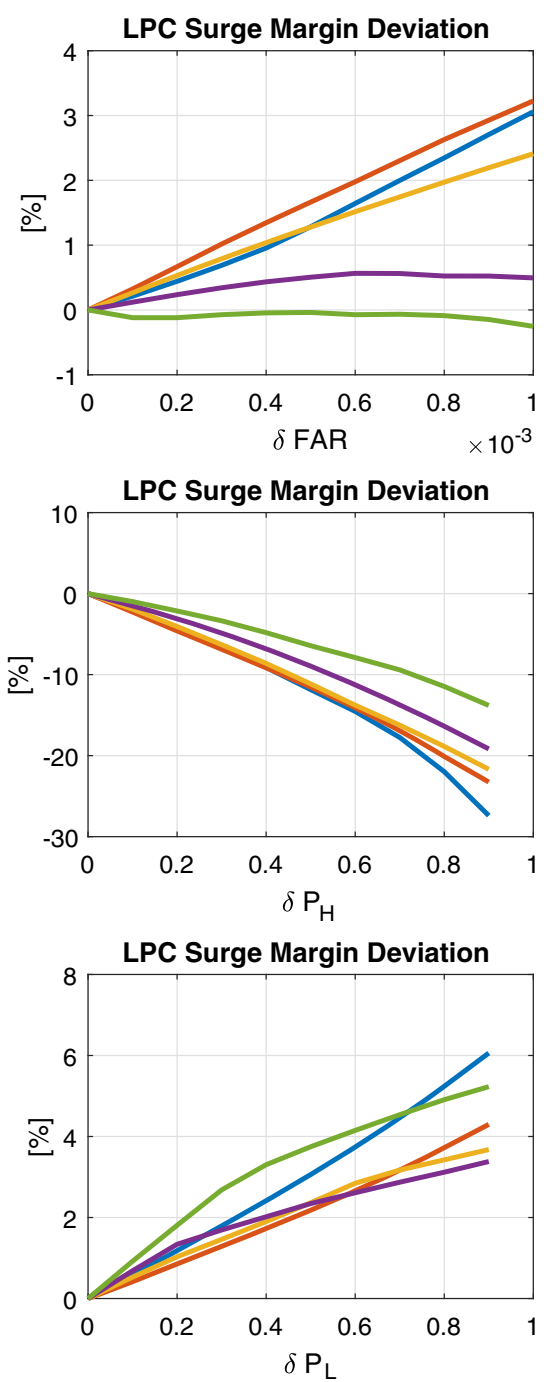
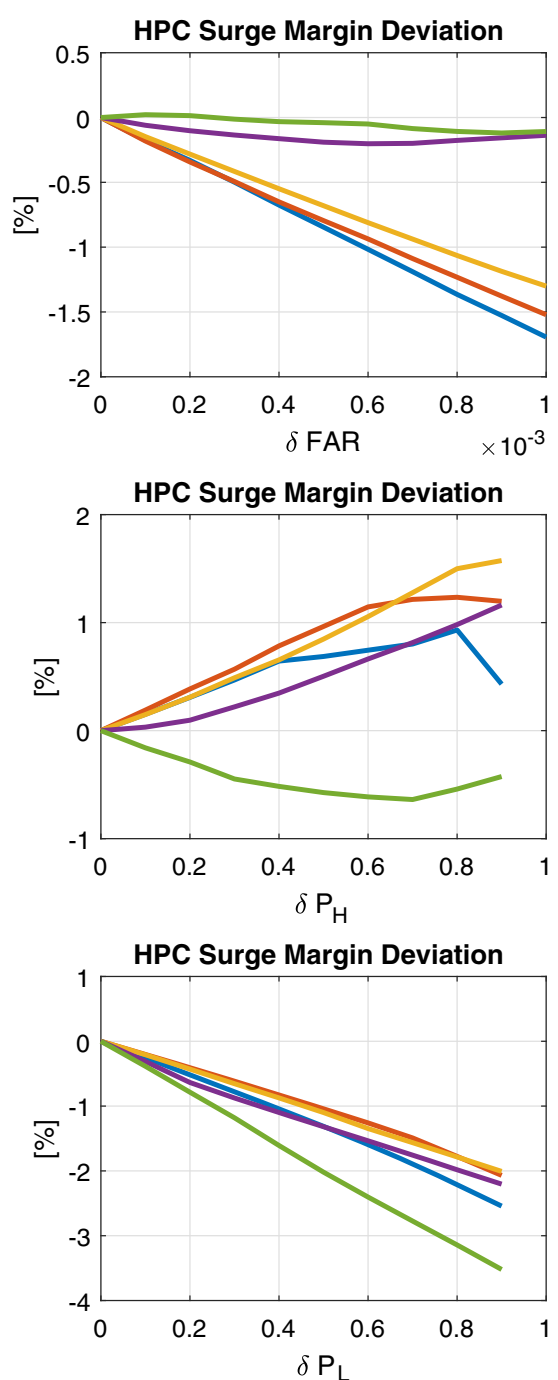

Fig. 3 Steady-state values of the nonlinear model for different operating points (thrusts). 
Thus, the inputs in Eq. (12) are $\delta \mathrm{FAR}, \delta P_{H \text { req }}, \delta P_{\text {Lreq }}$, and $P_{\text {jreq }}$; and the outputs in Eq. (12) are $\delta F_{g}, \delta P_{T}, \delta P_{D}, \delta P_{D}$, and $E_{j}$.

\section{Controller Design}

\section{A. Overall Architecture}

Our control architecture is shown in Fig. 4. The control system consists of a power split map and feedback controller designed as an MPC controller. The power split map determines the maximum and minimum optimal power differences $\left(P_{D \text { req }}\right.$ ax and $\left.P_{D \text { req }}{ }_{\min }\right)$ between the two generators as a function of the requested thrust level $F_{\text {greq }}$ and a total electrical power $P_{\text {Treq }}$ command. Then, the MPC controller generates the four control signals (FAR, $P_{H \text { req }}, P_{L \text { req }}$, and $P_{j \text { req }}$ ) to track the thrust, the total electrical power, and the optimal power difference setpoints while enforcing system constraints.

\section{B. Optimal Power Split}

In this section, the gas turbine engine behavior and operating regions are analyzed for different electrical power loads and operating points in steady state based on the models described in Sec. II. In particular, fuel consumption and compressor surge margins are considered.

In the previous work [30], the optimal power split map was based on a point that minimized fuel consumption for a given thrust and total electrical power output. In this paper, we generalize this approach and define the optimal power split range in which the fuel consumption deviates from the optimal fuel consumption by no more than $0.3 \%$. Examples of the fuel-optimal power split ranges obtained by numerical optimization applied to our model for thrust levels of 21,593, 27,593 and 32,593 lbf are shown in Fig. 5. The lines correspond to different levels of total electrical power, and they

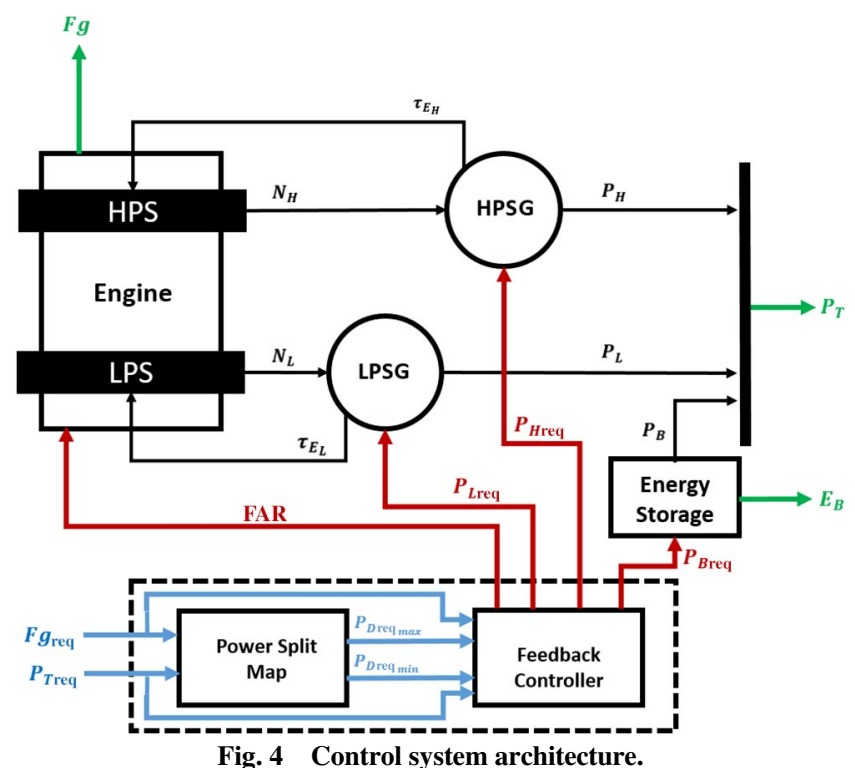

represent fuel consumption as a function of $P_{H}$ percentage for a given total electric power level. The black dotted lines indicate the optimal $P_{H}$ percentage where fuel consumption is minimal for given thrust and total electrical power output. The black solid lines indicate the interval of $P_{H}$ percentage values within which the fuel consumption is not worse than $0.3 \%$ of optimal; this interval changes, depending on the total electric power level and thrust. Thus, staying within the fuel-optimal split range (between the black solid lines) yields good fuel efficiency: that is, no more than $0.3 \%$ worse than that for the fueloptimal split line (black dotted lines).

As observed, when the total electrical power is small, the fueloptimal power split range is large: we have much control flexibility. However, when the total electrical power is large, the fuel-optimal power split range is small, and hence an accurate control strategy is necessary for fuel efficient operation at large electrical power levels.

The safe operation of the gas turbine engine also has to be ensured. Thus, an additional requirement to maintain sufficient fan, LPC, and HPC surge margins is considered in the definition of the power split range. Specifically, $15 \%$ as the minimum surge margin for the fan, $20 \%$ as the minimum surge margin for LPC, and $14 \%$ as the minimum surge margin for HPC are chosen for our control design and simulation-based case studies.

The surge margins as functions of $P_{H}$ percentage at different levels of thrust and electrical power are shown in Fig. $\underline{6}$. The black circles indicate the power split that yields the highest surge margin for the given thrust and electrical power output, and the black dotted lines indicate the surge margin lower bounds for each compressor. Thus, if the black circle lies below the black dotted line, it is impossible to satisfy the surge margin constraint for the given situation. Note that the fan always satisfies the lower limit, but LPC and HPC do not satisfy the lower limits for certain situations.

Note also that using the LPSG more increases the fan and LPC surge margins, and using the HPSG more increases the HPC surge margin. Furthermore, for some split ranges for the fan and LPC, the surge margins increase as the total electrical power output increases.

We now consider the power split ranges that satisfy both fuel efficiency and surge margin constraints for the given thrust and total electrical power level. See Fig. 7.

Not all values of $P_{H}$ percentage in the fuel-optimal power split range satisfy the surge margin limits. For instance, for 27,593 lbf of thrust and the total electrical power of $1.7 \mathrm{MW}$, the $P_{H}$ percentage of $40 \%$, as indicated by the cross, is within the fuel-optimal range, but it violates the HPC surge margin limit. The optimal power split range that takes into account the fuel efficiency constraints and surge margin limits is indicated in the shaded region of Fig. 7. The total electrical power output becomes more limited as the thrust increases, as expected. The optimal power split ranges for thrust varying between 21,593 and $32,593 \mathrm{lbf}$ and total electrical power varying between 0 and $3 \mathrm{MW}$ as indicated in Fig. 7 .

Note that, for a given thrust and total electrical power, the optimal power split range equivalently prescribes lower and upper bounds for the power difference $\left(P_{D}=P_{H}-P_{L}\right)$ between the HPSG and LPSG. Rather than using these values as constraints, in our MPC controller design, we choose to use both of these bounds $\left(P_{D \text { req } \min }\right.$ and $\left.P_{D \text { req }}{ }_{\max }\right)$,
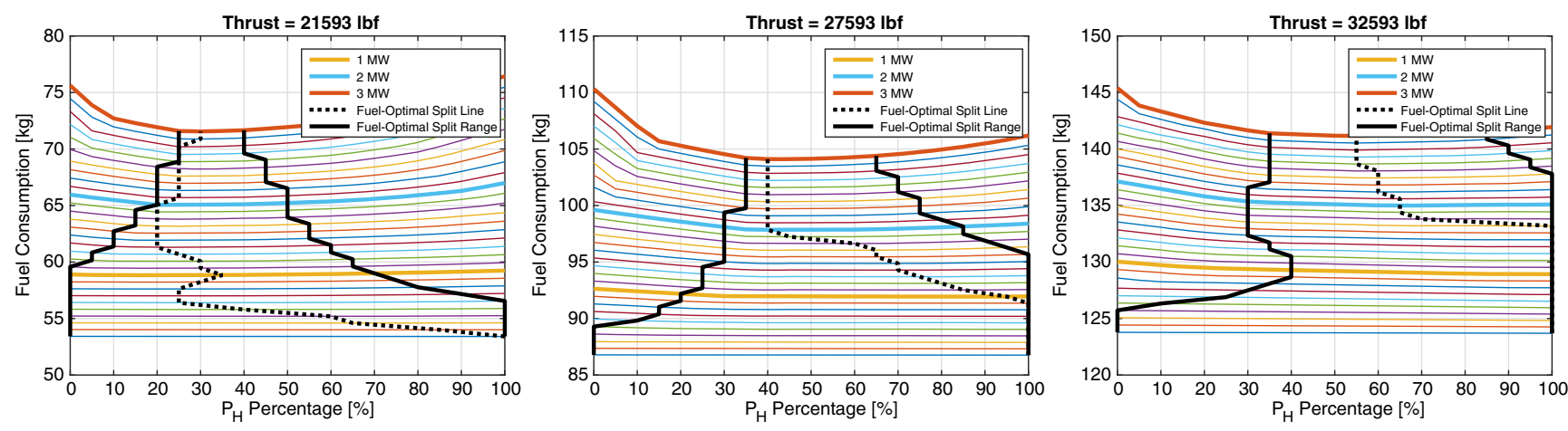

Fig. 5 Fuel-optimal power split range examples. 

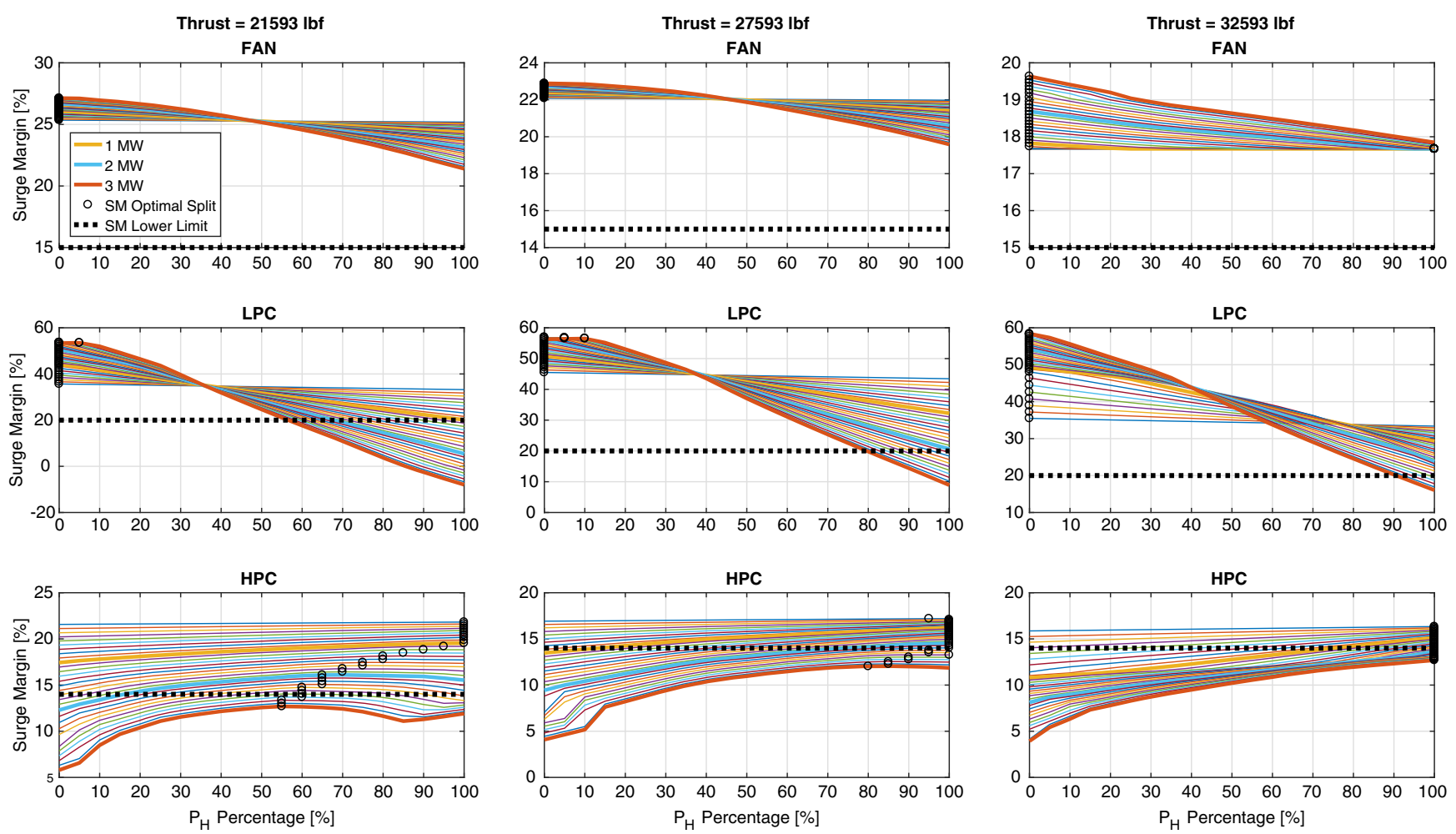

Fig. 6 Surge margin dependence on other variables.
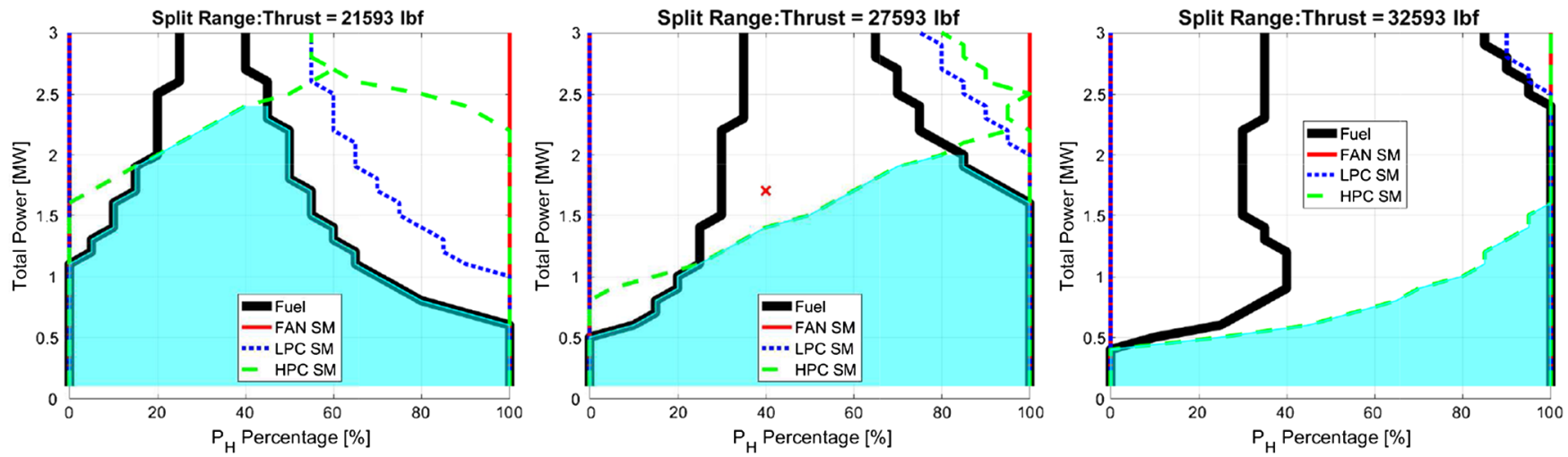

Fig. 7 Fuel and surge margin optimal power split ranges.

respectively, as setpoints in the cost function for $P_{D}$. As a result, $P_{D}$ is maintained in the range between these two setpoints as we have verified by simulations. This design approach leads to good performance.

\section{Energy Storage Elements Control Strategy}

The energy storage SOC is constrained between 40 and $60 \%$. These SOC constraints are treated as soft in the control design. The setpoint for the energy storage SOC is changed according to the following rule-based strategy:

1) When the thrust and load are decreased, track the high SOC setpoint, which is $90 \%$ in our simulation case study (charge).

2) When the thrust and load are increased, track the low SOC setpoint, which is $10 \%$ in our simulation case study (supply).

3) When one is decreased and the other is maintained, track the high SOC setpoint (charge).

4) When one is increased and the other is maintained, track the low SOC setpoint (supply).

5) For all other cases, track the setpoint corresponding to the midrange between the lower and upper limits (maintain desired SOC).

The basic idea behind these rules is to charge the energy storage if extra power is available and discharge the energy storage if extra power is needed. Given an SOC setpoint for the energy storage $j$ $\left(\mathrm{SOC}_{j_{d}}\right)$, the stored energy setpoint of the energy storage $j$ can be computed based on Eq. (4) as follows:

$$
E_{j_{d}}=\mathrm{SOC}_{j_{d}} \times E_{j_{\mathrm{Max}}}
$$

Thus, the stored energy setpoint in the MPC controller can be used instead of the SOC setpoint because the stored energy is one of the outputs of the linear model for our MPC controller design.

\section{Rate-Based MPC Controller Design}

\section{Scaled Model}

To alleviate the effects of different orders of magnitude of the inputs and outputs for the MPC controller, the inputs and outputs of the linear model are scaled before controller design. We want to scale the inputs and outputs such that the maximum value of each element in the scaled inputs and outputs is one.

Let $\delta u_{s}$ designate the vector of scaled inputs and $\delta u_{s_{\max }}$ be the maximum value of the scaled inputs so that each element in $\delta u_{s_{\max }}$ is one. Let the vector of the maximum values of the inputs $\delta u$ be given by $\delta u_{\max }=\left[\delta u_{1_{\max }} \delta u_{2_{\max }} \ldots \delta u_{i_{\max }}\right]^{T}$. Then, the relationship between the inputs and the scaled inputs is defined as 


$$
\delta u=S_{u} \delta u_{s}
$$

where $S_{u}$ is the input scaling matrix:

$$
S_{u}=\left[\begin{array}{cccc}
\delta u_{1_{\max }} & 0 & 0 & 0 \\
0 & \delta u_{2_{\max }} & 0 & 0 \\
0 & 0 & \ldots & 0 \\
0 & 0 & 0 & \delta u_{i_{\max }}
\end{array}\right]
$$

Let $\delta y_{s}$ be the vector of scaled outputs and $\delta y_{s_{\max }}$ be the maximum value of the scaled outputs so that each element in $\delta y_{s_{\max }}$ is one. Assume that the maximum value of the outputs is known. Let $\delta y$ be the outputs and $\delta y_{\max }=\left[\delta y_{1_{\max }} \delta y_{2_{\max }} \ldots \delta y_{j_{\max }}\right]^{T}$ be the maximum value of the outputs. Then, the relationship between the outputs and scaled outputs is defined as

$$
\delta y=S_{y} \delta y_{s}
$$

where $S_{y}$ is the output scaling matrix:

$$
S_{y}=\left[\begin{array}{cccc}
\delta y_{1_{\max }} & 0 & 0 & 0 \\
0 & \delta y_{2_{\max }} & 0 & 0 \\
0 & 0 & \ldots & 0 \\
0 & 0 & 0 & \delta y_{j_{\max }}
\end{array}\right]
$$

Assume that the unscaled linear system from Eq. (12) has the following form:

$$
\begin{aligned}
& \delta \dot{x}=A^{\prime \prime} \delta x+B^{\prime \prime} \delta u \\
& \delta y=C^{\prime \prime} \delta x+D^{\prime \prime} \delta u
\end{aligned}
$$

Substituting Eqs. (14) and (16) into Eq. (18) yields

$$
\begin{aligned}
\delta \dot{x} & =A^{\prime \prime} \delta x+B^{\prime \prime} S_{u} \delta u_{s} \\
S_{y} \delta y_{s} & =C^{\prime \prime} \delta x+D^{\prime \prime} S_{u} \delta u_{s}
\end{aligned}
$$

Then, the scaled system is

$$
\begin{aligned}
\delta \dot{x} & =A^{\prime \prime} \delta x+\hat{B} \delta u_{s} \\
\delta y_{s} & =\hat{C} \delta x+\hat{D} \delta u_{s}
\end{aligned}
$$

where $\hat{B}=B^{\prime \prime} S_{u}, \hat{C}=S_{y}^{-1} C^{\prime \prime}$, and $\hat{D}=S_{y}^{-1} D^{\prime \prime} S_{u}$. The model with the scaled inputs and outputs is used for control design.

\section{Offset State}

Before the rate-based model for MPC design is introduced, we describe how the nominal linear discrete-time model can be augmented with extra offset states to compensate for errors between linear model predictions and the response of the actual nonlinear system. The approach of compensating for model mismatch using offset states has also been used in other predictive control applications, such as for reference governors $[32,33]$. For the design of rate-based MPC, a linear discrete-time model is needed. Let the discrete-time linear model of the system have the following form:

$$
\begin{aligned}
\delta x_{k+1} & =A_{d}^{\prime} \delta x_{k}+B_{d}^{\prime} \delta u_{k}, \\
\delta y_{k} & =C_{d}^{\prime} \delta x_{k}+D_{d}^{\prime} \delta u_{k}
\end{aligned}
$$

where $k$ indicates discrete time instant, and $y_{k}$ denotes the output on which constraints are imposed. Suppose that the actual nonlinear system is given by

$$
\begin{aligned}
X_{k+1} & =f\left(X_{k}, U_{k}\right), \\
Y_{k} & =g\left(X_{k}\right)
\end{aligned}
$$

The offset state at the time instant $t$ is defined as follows:

$$
d_{t}=Y_{t}-\left(\delta y_{t}+y_{n o}\right)
$$

where $\delta y_{t}$ is the vector of outputs of the linearized model (deviations from the nominal values) at the time instant $t$, and $y_{n o}$ is the vector of nominal values of the output at which the model is linearized. We assume that the measurements or accurate estimates of $Y_{t}$ are available so that the current value of the offset state $d_{t}$ can be computed. Then, the linear prediction model is given by

$$
\begin{aligned}
\delta x_{k+1 \mid t} & =A_{d}^{\prime} \delta x_{k \mid t}+B_{d}^{\prime} \delta u_{k \mid t}, \\
d_{k+1 \mid t} & =d_{k \mid t}, \\
\delta y_{k \mid t} & =C_{d}^{\prime} \delta x_{k \mid t}+D_{d}^{\prime} \delta u_{k \mid t}+d_{k \mid t}
\end{aligned}
$$

where the standard notation in predictive control is used to designate predictions, e.g., $\delta x_{k \mid t}$ is the predicted state $k$ steps ahead when the prediction is made at the time instant $t$. In the sequel, this approach is used for handling surge constraints; hence, we assume (motivated by existing literature; see, e.g., [34]) that accurate estimates or measurements of surge margins are available in the gas turbine engine control strategy to be able to compute $d_{0 \mid t}=d_{t}$.

\section{Rate-Based MPC}

The design process of the rate-based MPC controller is now described. The states of the linear model used for prediction are assumed to be available from measurements and appropriately designed estimators. The rate-based MPC design described in this section is for the system configuration with a single energy storage element and two surge margin offset states. Other system configurations are handled similarly.

The discrete-time model is obtained using a sampling period of $0.04 \mathrm{~s}$ based on the scaled input-output model in Eq. (20). A rate-based MPC controller can be designed to perform set point tracking based on the discrete-time prediction model shown, without extra offset states, as

$$
\delta x_{k+1}=A_{d} \delta x_{k}+B_{d} \delta u_{k}, \quad \delta y_{k}=C_{d} \delta x_{k}+D_{d} \delta u_{k}
$$

where $A_{d}^{6 \times 6}, B_{d}^{6 \times 4}, C_{d}^{5 \times 6}, D_{d}^{5 \times 4}$, and $\delta y_{k}=\left[\begin{array}{lllll}\delta F_{g} & \delta P_{T} & \delta P_{D} & \delta P_{D} & E_{j}\end{array}\right]^{T}$. The control objective is to follow a requested command (setpoint) $r$ where $r=\left[\begin{array}{lllll}\delta F_{\text {greq }} & \delta P_{\text {Treq }} & \delta P_{D \text { req }_{\max }} & \delta P_{D \text { req }_{\min }} & E_{j d}\end{array}\right]^{T}$; that is, follow thrust requests, total electrical power requests, optimal maximum power difference requests, optimal minimum power difference requests, and stored energy requests, respectively. Then, the state and control increments are defined as

$$
\Delta x_{k}=\delta x_{k+1}-\delta x_{k}, \quad \Delta u_{k}=\delta u_{k+1}-\delta u_{k}
$$

and the error between the outputs $y_{k}$ and setpoints $r$ is defined as

$$
e_{k}=C_{d} \delta x_{k}+D_{d} \delta u_{k}-r
$$

Then,

$$
\begin{aligned}
\Delta x_{k+1} & =A_{d} \Delta x_{k}+B_{d} \Delta u_{k}, \\
e_{k+1} & =C_{d} \Delta x_{k}+D_{d} \Delta u_{k}+e_{k}, \\
\delta x_{k+1} & =\delta x_{k}+\Delta x_{k}, \\
\delta u_{k+1} & =\delta u_{k}+\Delta u_{k}
\end{aligned}
$$

Equation (28) can be extended with two surge margin offset states and two compensated surge margin states as described in Sec. III.D.2. The extended linear prediction model is as follows:

$$
\begin{aligned}
\Delta x_{k+1} & =A_{d} \Delta x_{k}+B_{d} \Delta u_{k}, \\
e_{k+1} & =C_{d} \Delta x_{k}+D_{d} \Delta u_{k}+e_{k}, \\
\delta x_{k+1} & =\delta x_{k}+\Delta x_{k}, \\
\delta u_{k+1} & =\delta u_{k}+\Delta u_{k}, \\
d_{k+1} & =d_{k}, \\
\delta \bar{x}_{k+1} & =F \delta x_{k+1}+d_{k+1}=F \delta x_{k}+F \Delta x_{k}+d_{k}
\end{aligned}
$$

where $d_{k}$ is the $2 \times 1$ surge margin offset states vector, $\delta \bar{x}_{k+1}$ is the $2 \times 1$ compensated surge margin deviations vector, and $F=\left[0_{2 \times 4} I_{2 \times 2}\right]$. The cost function to be minimized is given by 
Table 1 Agreement between the validation data and identified linear model at each operating point

\begin{tabular}{lcccccccccc}
\hline & \multicolumn{8}{c}{ Operating point determined by thrust level, lbf } \\
\cline { 2 - 10 } & 20,593 & 21,593 & 22,593 & 23,593 & 24,593 & 25,593 & 26,593 & 27,593 & 28,593 & 29,593 \\
\hline HPS speed & $94.11 \%$ & $91.89 \%$ & $92.48 \%$ & $88.54 \%$ & $82.82 \%$ & $82.19 \%$ & $83.38 \%$ & $81.34 \%$ & $80.60 \%$ & $89.54 \%$ \\
LPS speed & $92.66 \%$ & $92.73 \%$ & $82.14 \%$ & $80.39 \%$ & $79.00 \%$ & $83.50 \%$ & $81.36 \%$ & $80.24 \%$ & $82.32 \%$ & $87.54 \%$ \\
Thrust & $93.72 \%$ & $93.21 \%$ & $89.14 \%$ & $84.78 \%$ & $87.11 \%$ & $82.69 \%$ & $83.00 \%$ & $81.41 \%$ & 82.10 & $88.87 \%$ \\
LPC surge margin & $90.44 \%$ & $86.29 \%$ & $84.46 \%$ & $76.60 \%$ & $72.83 \%$ & $64.32 \%$ & $64.97 \%$ & $63.80 \%$ & $69.89 \%$ & $79.59 \%$ \\
HPC surge margin & $90.02 \%$ & $92.43 \%$ & $52.71 \%$ & $42.85 \%$ & $51.72 \%$ & $68.44 \%$ & $40.84 \%$ & $82.62 \%$ & $43.69 \%$ & $89.94 \%$ \\
Average & $92.99 \%$ & $91.31 \%$ & $80.19 \%$ & $74.63 \%$ & $74.70 \%$ & $76.23 \%$ & $71.11 \%$ & $77.88 \%$ & $71.72 \%$ & $87.09 \%$ \\
\hline \hline
\end{tabular}

Table 2 Constraints for LQR and MPC controllers

\begin{tabular}{|c|c|c|}
\hline & $\mathrm{QR}^{\mathrm{a}}$ & MPC \\
\hline \multicolumn{3}{|c|}{ Input constraints } \\
\hline FAR & N/A & $\pm \inf$ \\
\hline$P_{H}, \mathrm{~kW}$ & N/A & $\leq$ \\
\hline & N/A & $0 \leq$ \\
\hline$\dot{F} A R, / \mathrm{s}$ & N/A & $\pm 0 . \overline{0}$ \\
\hline$\dot{P}_{H}, \mathrm{~kW} / \mathrm{s}$ & N/A & \pm 100 \\
\hline$\dot{P}_{L}, \mathrm{~kW} / \mathrm{s}$ & N/A & \pm 1000 \\
\hline \multicolumn{3}{|c|}{ State constraints } \\
\hline $\mathrm{SM}_{\mathrm{FAN}}, \%$ & N/A & $15 \leq$ \\
\hline $\mathrm{SM}_{\mathrm{LPC}}, \%$ & N/A & $20 \leq$ \\
\hline $\mathrm{SM}_{\mathrm{HPC}}, \%$ & N/A & $14 \leq$ \\
\hline
\end{tabular}

aN/A denotes "not applicable."

$$
J_{N}=\sum_{k=0}^{N-1} e_{k \mid t}^{T} Q e_{k \mid t}+\Delta u_{k \mid t}^{T} R \Delta u_{k \mid t},
$$

subject to the constraints

$$
\begin{aligned}
& \delta x_{\min } \leq \delta x_{k \mid t} \leq \delta x_{\max }, \quad k=0, \ldots, N, \\
& \delta u_{\min } \leq \delta u_{k \mid t} \leq \delta u_{\max }, \quad k=0, \ldots, N-1, \\
& \Delta u_{\min } \leq \Delta u_{k \mid t} \leq \Delta u_{\max }, \quad k=0, \ldots, N-1
\end{aligned}
$$

where $N$ is the prediction horizon; $Q$ is a $5 \times 5$ diagonal weight matrix associated with the five errors; $R$ is a $4 \times 4$ diagonal weight matrix associated with the four inputs; $e_{k \mid t}$ is the predicted error $k$ steps ahead when the prediction is made at time instant $t ; \delta u_{k \mid t}$ is the predicted input $k$ steps ahead when the prediction is made at time instant $t ; \delta x_{\min }$ and $\delta x_{\max }$ designate state bounds; and $\delta u_{\min }, \delta u_{\max }, \Delta u_{\min }$, and $\Delta u_{\max }$ designate the bounds on the control inputs and their time rates of change.
Note that the cost function is constructed to penalize the deviation of power difference between the two generators $P_{D}$ from the maximum power difference setpoint $P_{D \text { req }}$ and the minimum power difference setpoint $P_{D \text { req }}$, where these setpoints are computed from optimal power split ranges. The same weights are used for both tracking errors. This strategy maintains $P_{D}$ in between the two setpoints, and hence within/in the middle of the optimal power split range.

The aforementioned tracking MPC formulation can be rewritten as a standard MPC problem (to which standard MPC solvers are applicable) for an extended system with a larger state vector:

$$
x_{k \mid t}^{\mathrm{ext}}=\left[\begin{array}{llllll}
\Delta x_{k \mid t}^{T} & e_{k \mid t}^{T} & \delta x_{k \mid t}^{T} & \delta u_{k \mid t}^{T} & d_{k \mid t}^{T} & \delta \bar{x}_{k \mid t}^{T}
\end{array}\right]^{T}
$$

and the extended state prediction model given by

$$
x_{k+1 \mid t}^{\mathrm{ext}}=\left[\begin{array}{cccccc}
A_{d} & 0 & 0 & 0 & 0 & 0 \\
C_{d} & I_{5 \times 5} & 0 & 0 & 0 & 0 \\
I_{6 \times 6} & 0 & I_{6 \times 6} & 0 & 0 & 0 \\
0 & 0 & 0 & I_{4 \times 4} & 0 & 0 \\
0 & 0 & 0 & 0 & I_{2 \times 2} & 0 \\
F & 0 & F & 0 & I_{2 \times 2} & 0
\end{array}\right] x_{k \mid t}^{\mathrm{ext}}+\left[\begin{array}{c}
B_{d} \\
D_{d} \\
0 \\
I_{4 \times 4} \\
0 \\
0
\end{array}\right] \Delta u_{k \mid t}
$$

For this extended system, the state penalty matrix has the form

$$
Q_{\text {ext }}=\left[\begin{array}{cccccc}
0 & 0 & 0 & 0 & 0 & 0 \\
0 & Q & 0 & 0 & 0 & 0 \\
0 & 0 & 0 & 0 & 0 & 0 \\
0 & 0 & 0 & 0 & 0 & 0 \\
0 & 0 & 0 & 0 & 0 & 0 \\
0 & 0 & 0 & 0 & 0 & 0
\end{array}\right]
$$

\section{JT9D Dynamic Gas Turbine Engine and Electrical System Simulation}

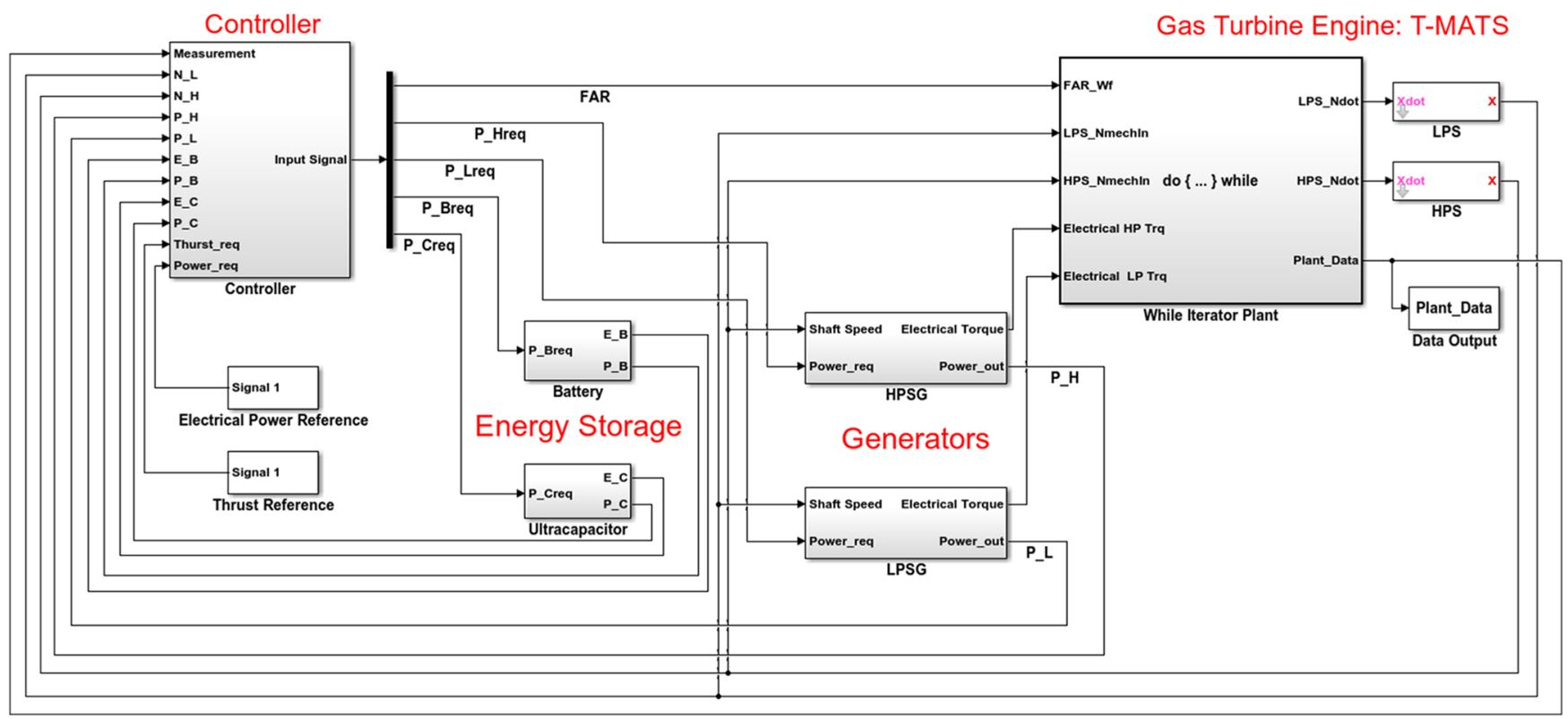


Table 3 Parameters for LQR and MPC controllers (Inf, infinity)

\begin{tabular}{lccc}
\hline \hline & Uncoordinated & Integrated & Integrated \\
& LQR & LQR & MPC \\
\hline Sampling time, s & 0.04 & 0.04 & 0.04 \\
Prediction horizon, steps & Inf & Inf & 30 \\
Constraint horizon, & N/A & N/A & 30 \\
steps & & & \\
Control horizon, steps & Inf & Inf & 10 \\
\hline \hline
\end{tabular}

and the control penalty matrix is $R_{\text {ext }}=R$. Two choices of prediction horizon and sampling period are considered: $N=100$ with a sampling period of $0.04 \mathrm{~s}$ (which corresponds to $4 \mathrm{~s}$ of prediction), and $N=30$ with a sampling period of $0.12 \mathrm{~s}$ (which corresponds to
$3.6 \mathrm{~s}$ of prediction). The MPT [16] is used to implement and simulate our MPC controller. Hard constraints are imposed on the power output of the generators to be positive and power to/from the energy storage elements. Soft constraints are imposed on surge margins and stored energy of the energy storage elements.

\section{E. Multiple MPC Controllers}

The rate-based MPC controller exploits a single linear model obtained as a linearization of the nonlinear model at 27,593 lbf of thrust and zero electrical load. If the system operates far from this nominal operating point, model inaccuracies may lead to poor closed-loop performance. The standard approach to address this issue [35-37], sometimes called switched MPC, is to design a set of linear $\mathrm{MPC}$ controllers based on linear models at several operating points,
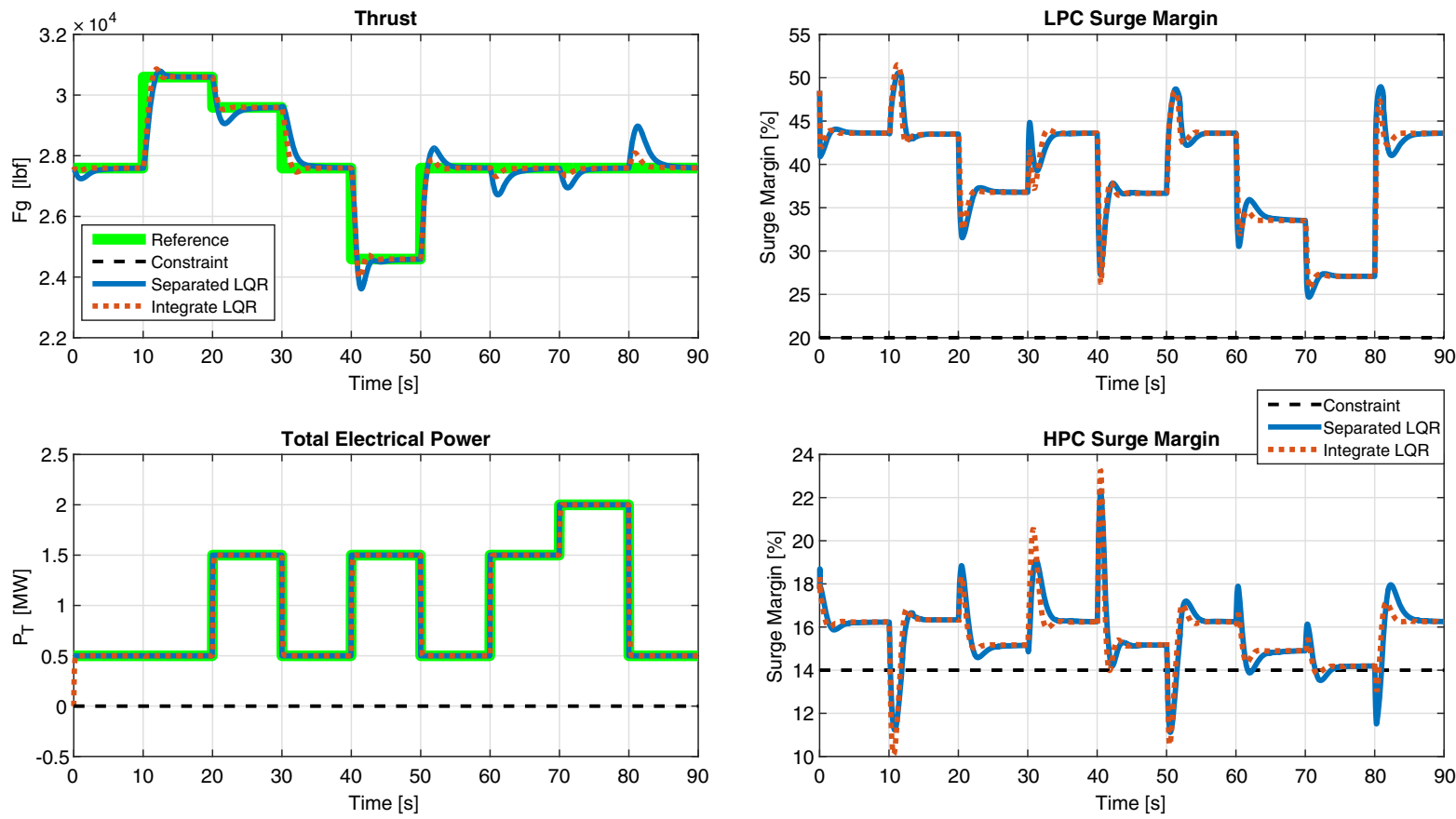

Fig. 9 Comparison of uncoordinated LQR and integrated LQR controllers.
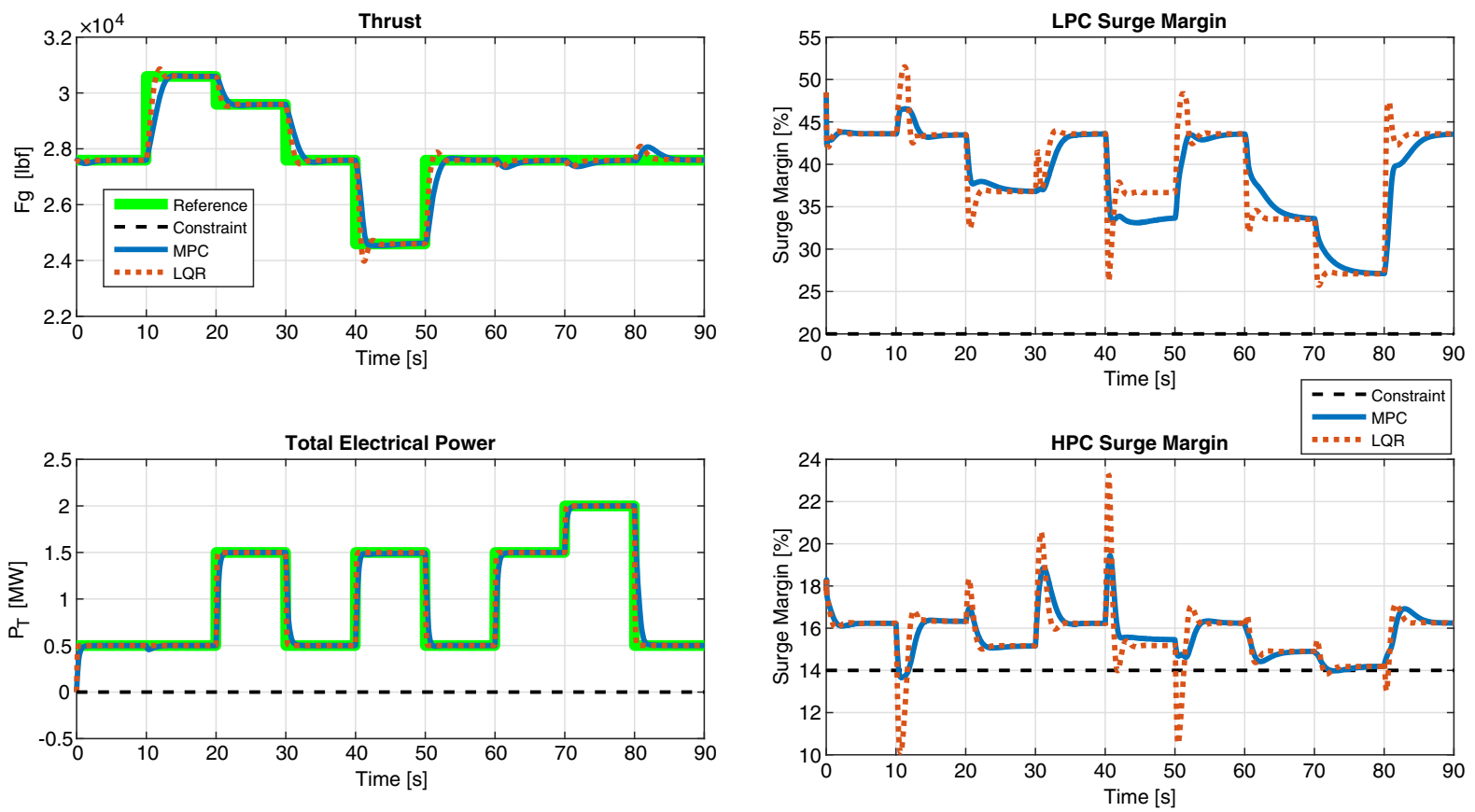

Fig. 10 Comparison of integrated LQR and MPC controllers. 

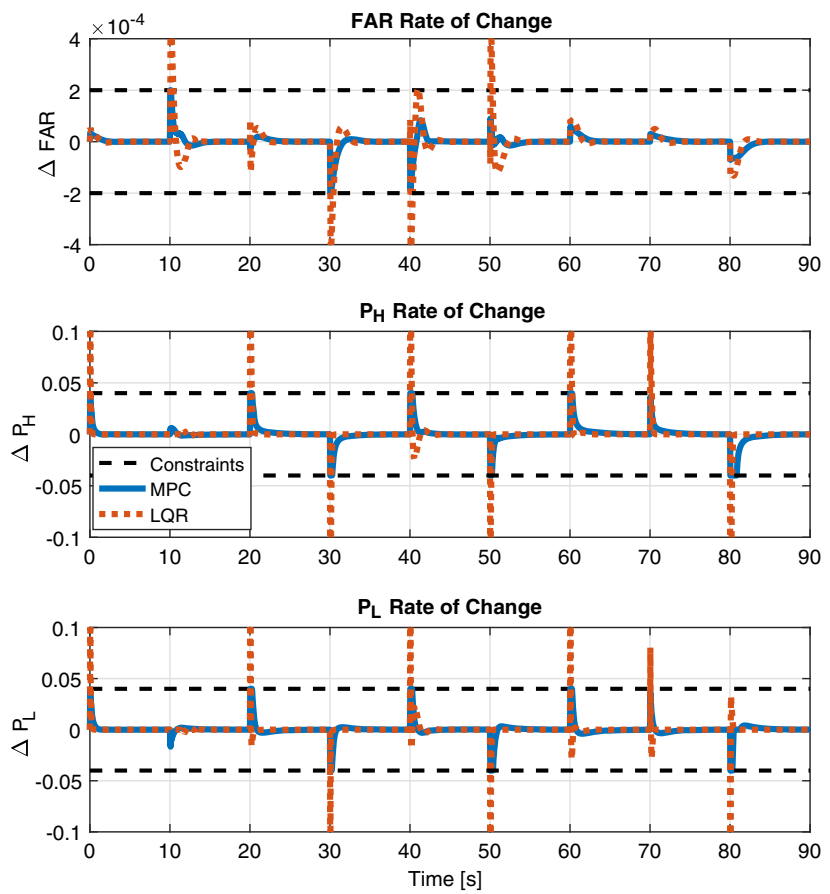

Fig. 11 Comparison of integrated LQR and MPC controllers with respect to input constraint handling.

and then switch between the corresponding MPC controllers depending, in our case, on the engine thrust level. The switching process can be summarized as follows:

1) If the current operating point is different from the previous operating point, go to step 3. Otherwise, go to step 2.

2) Generate control input using the current controller; then, return to step 1 .

3) Switch the controller and initialize the previous linear state as follows:

$$
\delta x_{\text {old }}=0
$$

4) Update the previous input as follows:

$$
\delta u_{\text {old }}=\delta u_{\text {old }}-\left(u_{n 0}-u_{\text {old } 0}\right)
$$

where $u_{n 0}$ is the nominal input at the new operating point, and $u_{\text {old } 0}$ is the nominal input at the previous operating point.

5) Update the current linear state as follows:

$$
\delta x=A_{d} \delta x_{\text {old }}+B_{d} \delta u_{\text {old }}
$$

where $A_{d}$ and $B_{d}$ are the discrete linear system matrices at the new operating point.

6) Compute the input for the MPC controller as follows:

$$
x_{\text {cont }}=\left[\begin{array}{llllll}
\left(\delta x-\delta x_{\text {old }}\right)^{T} & e^{T} & \delta x^{T} & \delta u_{\text {old }}^{T} & d^{T} & \delta x^{T}+d^{T}
\end{array}\right]^{T}
$$

where $e$ is the measured error, and $d$ is the vector of offset states. Then, generate the control input using the current controller and return to step 1 .
Our switching MPC design was based on 10 operating points corresponding to thrust levels of 20,593, 21,593, 22,593, 23,593, 24,593, 25,593, 26,593, 27,593, 28,593, and 29,593 lbf. At each operating point, the linearized model was generated using identification techniques as in Sec. II.D.

The agreements between the validation data and identified linear models have been computed for each operating point as described in Sec. II.D.1. See Table 1. Because the LPC and HPC surge margin behavior is highly nonlinear, as shown in Fig. 3 , the LPC and HPC surge margins agreements are relatively poor when compared to the other agreements. However, these inaccuracies can be handled by using the extra offset state as described in Sec. III.D.2. The 10 MPC controllers used in the switched MPC design are generated based on these linearized models and the same weights.

\section{Simulations and Results}

The results of different simulation case studies are reported in this section. First, uncoordinated linear quadratic regulators, integrated LQRs, and integrated MPCs are compared to show the benefits of the integrated control and of the MPC. Second, the responses with different energy storage elements are compared, and the benefits of adding energy storage elements to the system are illustrated. Third, LQR, MPC, MPC with surge margin offset states (offset MPC), and 10 MPC [multiple MPC (MMPC)] control designs are compared. The MPC controllers with offset states for the systems configurations with and without energy storage elements are also compared.

The control objective is to satisfy the surge margin constraints, maintain the requested thrust level, and supply the requested electrical power during a $90 \mathrm{~s}$ simulation. All the simulations start from steady state with $F g=27,593 \mathrm{lbf}$, LPC surge margin $=44.7 \%, \mathrm{HPC}$ surge margin $=17.3 \%, \mathrm{FAR}=0.0187$, and $P_{H \text { req }}=P_{L \text { req }}=P_{\text {jreq }}=P_{T}=0 \mathrm{MW}$. The initial energy storage SOC is $50 \%$, and the desired SOC range is between 40 and $60 \%$. The state and input constraints are summarized in Table 2 . Because LQR controllers do not enforce constraints, no constraints are defined for them.

The charge/discharge rate constraints of the energy storage elements vary based on their types, so these constraints are as indicated for each simulation.

All simulations are performed on the fully nonlinear model of the system. The Simulink model of the closed-loop system with the offset MPC controller and the energy storage elements is shown in Fig. 8 .

\section{A. Performance Metrics}

Performance metrics have been defined to compare different controllers. The first metric is the average thrust deviation from the set point $F g_{\text {AvgDev }}$, which reflects the thrust tracking performance and is defined by

$$
F g_{\text {AvgDev }}=\frac{\sum\left|F g_{\text {ref }}-F g\right|}{n_{t}} \quad \text { or } \quad \frac{\int_{0}^{t_{d}}\left|F g_{\text {ref }}-F g\right|}{t_{d}}
$$

where $F g_{\text {ref }}$ is the thrust setpoint, $F g$ is the thrust, $n_{t}$ is the number of samples, and $t_{d}$ is the total simulation duration. A smaller value of $F g_{\text {AvgDev }}$ indicates better thrust request tracking.

The second metric is the average total electrical power deviation from the setpoint $P_{T_{\text {Avgev }}}$, which reflects the performance in supplying the requested total electrical power. This metric is defined as

Table 4 Performance comparison of uncoordinated LQR, integrated LQR, and integrated MPC

\begin{tabular}{lccc}
\hline \hline & Uncoordinated LQR & Integrated LQR & Integrated MPC \\
\hline$W_{f}, \mathrm{~kg}$ & 84.74 & 84.77 & 84.59 \\
$F g_{\text {AvgDev }}, \mathrm{lbf}$ & 236.13 & 109.05 & 191.79 \\
$P_{T_{\text {AvgDev }}, \mathrm{kW}}$ & 0 & 7.42 & 32.48 \\
$n_{\text {smv }}$, times & 5 & 5 & 2 \\
$t_{d_{\text {smv }}, \mathrm{s}}$ & $1.6 / 1.36 / 0.68 / 2.28 / 0.92$ & $1.44 / 0.12 / 1.16 / 1.16 / 0.48$ & $1.36 / 1.32$ \\
$\mathrm{SM}_{\text {Max }}, \%$ & $2.78 / 2.89 / 0.13 / 0.48 / 2.49$ & $3.90 / 0.02 / 3.47 / 0.17 / 0.97$ & $0.34 / 0.03$ \\
\hline \hline
\end{tabular}



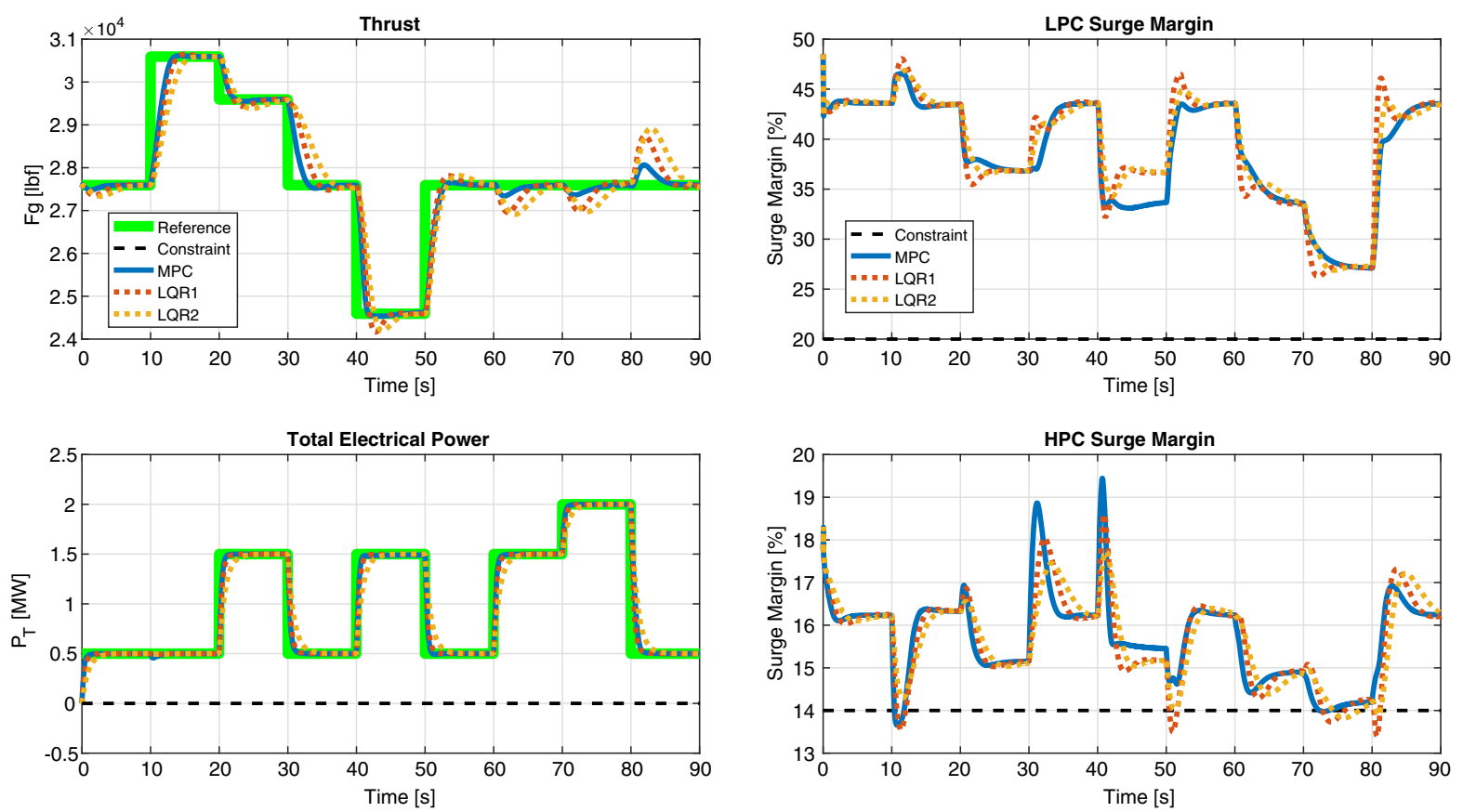

Fig. 12 Comparison of integrated LQR and MPC controllers.

$$
P_{T_{\mathrm{AvgDev}}}=\frac{\sum\left|P_{T_{\mathrm{ref}}}-P_{T}\right|}{n_{t}} \quad \text { or } \quad \frac{\int_{0}^{t_{d}}\left|P_{T_{\mathrm{ref}}}-P_{T}\right|}{t_{d}}
$$

where $P_{T_{\text {ref }}}$ is the total electrical power setpoint (i.e., the sum of required electrical loads), and $P_{T}$ is the total electrical power generated

Table 5 Specification of battery cell and ultracapacitor cell

\begin{tabular}{lcc}
\hline \hline & AMP20 battery cell & K2 ultracapacitor cell \\
\hline Weight, g & 496 & 520 \\
Stored energy, nominal, $\mathrm{W} \cdot \mathrm{h}$ & 65 & 4 \\
Discharge power, nominal, $\mathrm{kW}$ & 1.2 & 4.4 (maximum 9.4) \\
Voltage, nominal, $\mathrm{V}$ & 3.3 & 2.85 \\
\hline \hline
\end{tabular}

by the system. A smaller value of $P_{T_{\text {AvgDev }}}$ indicates better total electrical power tracking, i.e., better supply of the electrical loads.

The next set of metrics is introduced to characterize the surge margin (SM) violations. The metrics are as follows: the number of surge margin violations $n_{\mathrm{smv}}$, the duration of the $i$ th violation $t_{d_{\mathrm{smv}}}^{i}$, and the maximum amount of the $i$ th violation $\mathrm{SM}_{\mathrm{Max} V}^{i}$.

The final metric is the total fuel consumption $w_{f}$. A smaller value of $w_{f}$ indicates better fuel efficiency.

\section{B. Comparison of Uncoordinated and Coordinated Control}

In this section, three different controllers are compared: uncoordinated LQR, integrated LQR, and integrated MPC. For the purpose of this comparison, the system without the energy storage

Table 6 Specifications of three different energy storage element configurations

\begin{tabular}{lcccc}
\hline \hline & & & \multicolumn{2}{c}{$\begin{array}{c}\text { Battery-ultracapacitor pack } \\
\text { (BAT-UCAP pack) }\end{array}$} \\
\cline { 2 - 5 } & Battery pack (BAT pack) & Ultracapacitor-pack (UCAP pack) & Battery pack & Ultracapacitor pack \\
\hline Number of cells & 77 & 88 & 38 & 44 \\
Weight, kg & 39 & 46 & 19 & 23 \\
Volume, liter & 20.3 & 36 & 10 & 18 \\
Stored energy, nominal, $\mathrm{kW} \cdot \mathrm{h}$ & 5 & 0.35 & 2.4 & 0.176 \\
Voltage, nominal, V & $\approx 250$ & $\approx 250$ & $\approx 125$ & $\approx 125$ \\
Discharge rate, nominal, $\mathrm{kW}$ & 92 & 389 (maximum 824) & 45 & 193 (maximum 413) \\
\hline
\end{tabular}

Table 7 Parameters and energy storage element input constraints for MPC controllers

\begin{tabular}{lccccc}
\hline \hline & \multicolumn{5}{c}{ MPC } \\
\cline { 2 - 6 } & \multicolumn{7}{c}{ BAT-UCAP pack } \\
\cline { 2 - 6 } & Without & BAT pack & UCAP pack & BAT pack & UCAP pack \\
\hline Sampling time, s & 0.04 & 0.04 & 0.04 & 0.04 & 0.04 \\
Prediction horizon, steps & 100 & 100 & 100 & 100 & 100 \\
Constraints horizon, steps & 100 & 100 & 100 & 100 & 100 \\
Control horizon, steps & 30 & 30 & 30 & 30 & 30 \\
& & Input constraints & & & \\
$P_{j}, \mathrm{~kW}$ & N/A & \pm 100 & \pm 800 & \pm 50 & \pm 400 \\
$P_{j}, \mathrm{~kW} / \mathrm{s}$ & N/A & \pm inf & \pm inf & \pm inf & \pm inf \\
\hline \hline
\end{tabular}



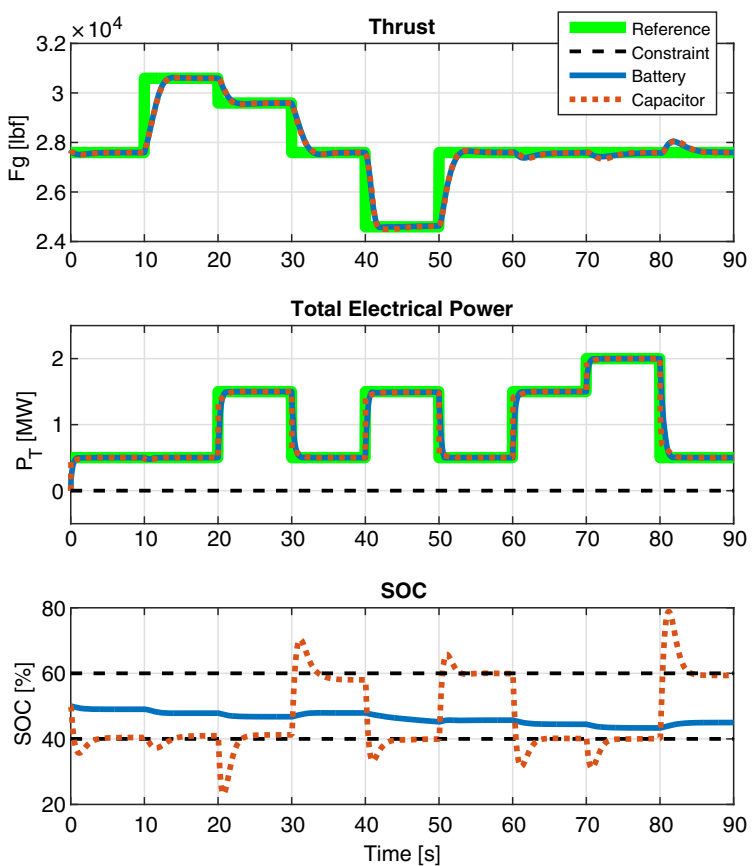

Fig. 13 Comparison of battery and ultracapacitor packs.

elements is considered. The uncoordinated LQR controller only adjusts the FAR for the engine, whereas the generator power requests are managed according to a simple strategy of the form

$$
\begin{aligned}
P_{\text {Hreq }} & =\frac{P_{\text {Treq }}+P_{D \text { req }}}{2}, \\
P_{\text {Lreq }} & =\frac{P_{\text {Treq }}-P_{D \text { req }}}{2}
\end{aligned}
$$

In the integrated LQR and MPC controllers, a single controller is used for the whole system to provide coordinated control of three inputs. Each choice of the controller was tuned for best performance. The controller parameters are shown in Table 3 .

The simulation results of the uncoordinated LQR and integrated LQR controllers are shown in Fig. 9. As shown in the left subfigures, both controllers accurately track the total electrical power setpoint. However, the uncoordinated LQR controller yields larger thrust deviations when the step change of electrical power occurs because it does not account for the interactions between the generators and the gas turbine engine. As expected, both controllers violate surge margin constraints.

The simulation results of the integrated LQR and integrated MPC controllers are shown in Fig. 10. As shown in the right subfigures, the integrated MPC controller satisfies the soft surge margin constraints, except for a few small violations, whereas the integrated LQR controller does not. Note that the tracking of thrust and total electrical power is worse for the integrated MPC than for the integrated LQR controller; however, unlike the LQR, the former enforces the constraints; see Fig. 11.

The performance metrics for the three controllers are compared in Table 4. The uncoordinated LQR controller yields the best electrical power tracking performance but the worst thrust tracking performance. The integrated LQR controller yields the best thrust tracking performance and good electrical power tracking performance. However, both LQR controllers violate the HPC surge margin constraint five times, sometimes by a large amount $(2.89 \%$ for the uncoordinated LQR and $3.9 \%$ for the integrated LQR at time instants of 50.52 and $10.68 \mathrm{~s}$, respectively), whereas the integrated MPC controller only violates these constraints twice by very small amounts.

To illustrate the advantages of MPC over the LQR, note that tuning the LQR controller less aggressively could remove the surge margin violations but, at the same time, the thrust and total electrical power tracking will be slower for all the transients, even for small transients,
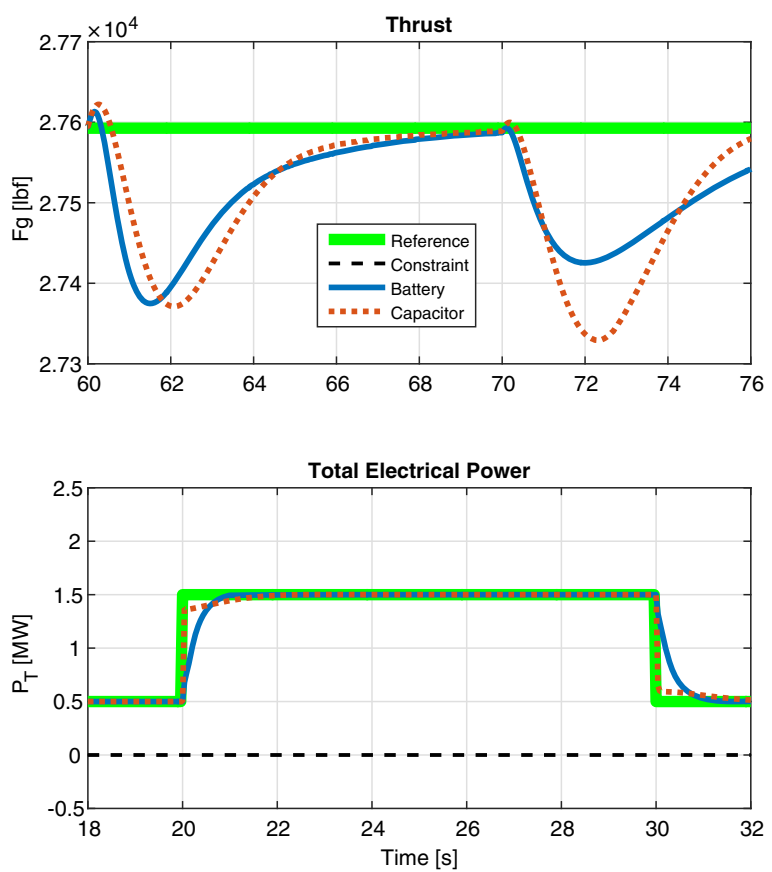

for which there is no danger of surge margin violations. Examples of different tunings of the integrated LQR controller and comparison with the integrated MPC are shown in Fig. 12.

As indicated in the figures, tuning the integrated $\mathrm{LQR}$ controller less aggressively, indicated by LQR 1 in Fig. 12, reduces the surge margin violations. However, it stills yields more surge margin violations and, furthermore, worse thrust tracking performance than the integrated MPC. If the integrated LQR controller is tuned further (less aggressively), as indicated by LQR2 in the figures, most of the surge margin violations disappear, but the thrust and total electrical power tracking are poor. Meanwhile, the MPC controller can provide aggressive thrust and total electrical power tracking when there is no danger of surge margin constraint violation, as well as less aggressive tracking when the surge margin constraints are active. Furthermore, in addition to the surge margins, there are other constraints handled by the MPC controller (e.g., positive power limit, charge and discharge rate of the energy storage elements, etc.) that the LQR controller is not designed to handle. In the subsequent sections, the uncoordinated LQR controller design is omitted, and only the integrated controllers are considered.

\section{Comparison of Different Energy Storage Elements}

In this section, the system responses with different energy storage elements are compared, and the benefits of adding energy storage elements are illustrated. Batteries and ultracapacitors are chosen as the energy storage elements. The specifications of the chosen battery cells [38] and ultracapacitor cells [39] are listed in Table $\underline{5}$.

Based on the specifications, three different energy storage element configurations are considered: a battery pack, an ultracapacitor pack, and a battery-ultracapacitor pack. All the energy storage elements are limited to less than $50 \mathrm{~kg}$ and $40 \mathrm{~L}$ when considering the limited space

Table 8 Performance comparison of the systems without energy storage and with different energy storage elements

\begin{tabular}{lcccc}
\hline \hline & \multicolumn{4}{c}{ MPC } \\
\cline { 2 - 5 } & Without & BAT pack & UCAP pack & BAT-UCAP pack \\
\hline$W_{f}, \mathrm{~kg}$ & 84.59 & 84.56 & 84.6 & 84.57 \\
$F g_{\text {AvgDev }}, \mathrm{lbf}$ & 191.79 & 187.17 & 191.87 & 188.84 \\
$P_{T_{\text {AvgDev }}, \mathrm{kW}}$ & 32.48 & 26.69 & 15.02 & 19.32 \\
$n_{\text {smv }}$, times & 2 & 1 & 2 & 2 \\
$t_{d_{\text {smv }},}, \mathrm{s}$ & $1.36 / 1.32$ & 1.32 & $1.36 / 1.72$ & $1.32 / 0.76$ \\
$\mathrm{SM}_{\text {Max }}$ & $0.34 / 0.03$ & 0.34 & $0.34 / 0.06$ & $0.34 / 0.01$ \\
\hline \hline
\end{tabular}



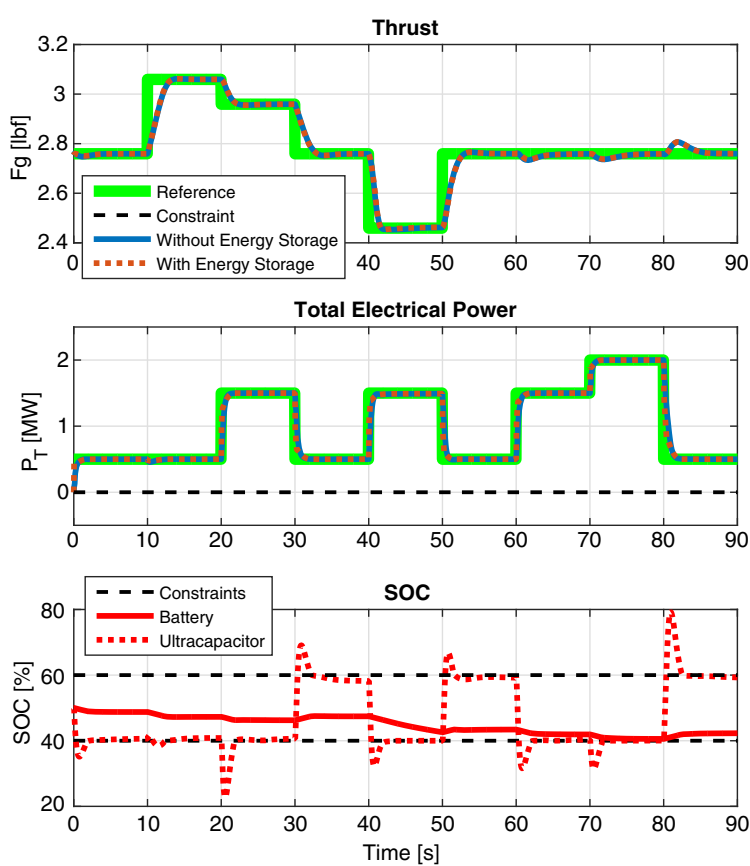

Fig. 14 Comparison of controller performance without and with energy storage elements.

on aircraft. Note that, currently, relatively small stored energy is considered compared to our electrical load requests. The specifications of the energy storage elements are shown in Table 6.

There are clearly differences between the battery pack and the ultracapacitor pack in terms of the stored energy and the discharge rate. The battery pack has much larger stored energy than the ultracapacitor pack, but the ultracapacitor pack has a much faster discharge rate than the battery pack. The battery-ultracapacitor packs can take advantage of both characteristics. Based on the specifications in Table 6 , the controller parameters and the energy storage element input constraints (charge/discharge rate constraints) are defined as shown in Table 7.

All of the MPC controllers designed for systems with three different storage element configurations use the same controller parameters and constraints, except for the constraints on $P_{j}$ that are determined based on the discharge rate of the energy storage elements. Constraints on $\dot{P}_{j}$ are not considered. The simulation results of MPC with the battery pack and the ultracapacitor pack are shown in Fig. 13.

There are clear differences in the responses observed for the two types of energy storage elements. The SOC of the ultracapacitor pack varies more than the SOC of the battery pack because the ultracapacitor pack has much smaller stored energy than the battery pack, whereas the ultracapacitor pack is much faster than the battery pack, so it can supply the electrical loads very quickly; see the rightbottom subfigure, which shows the time history of the total electrical power in the time interval between 18 and $32 \mathrm{~s}$. However, due to limited stored energy, the ultracapacitor cannot supply the electrical loads for a long time; instead, it needs to be recharged to recover a SOC in the $40-60 \%$ range, as shown in the left-bottom SOC subfigure in the time interval between 20 and $25 \mathrm{~s}$. Meanwhile, the battery pack allows for better thrust command tracking, as shown in the right-upper subfigure in Fig. 13, which represents the trajectory of thrust in the time interval between 60 and $75 \mathrm{~s}$. This is reasonable, given the large stored energy in the battery pack. The battery pack can deliver electrical power for a long time, which helps the gas turbine engine to use power for thrust generation instead of supplying the generators to satisfy the loads. In addition, the battery pack reduces surge margin violations, as shown in Table $\underline{8}$. Thus, for faster electrical loads supplying, the ultracapacitor pack appears to be a suitable energy storage choice; however, for faster thrust responses and stable gas turbine engine operation, the battery pack is preferred.

We next compare cases without and with the batteryultracapacitor pack that take advantage of the characteristics of
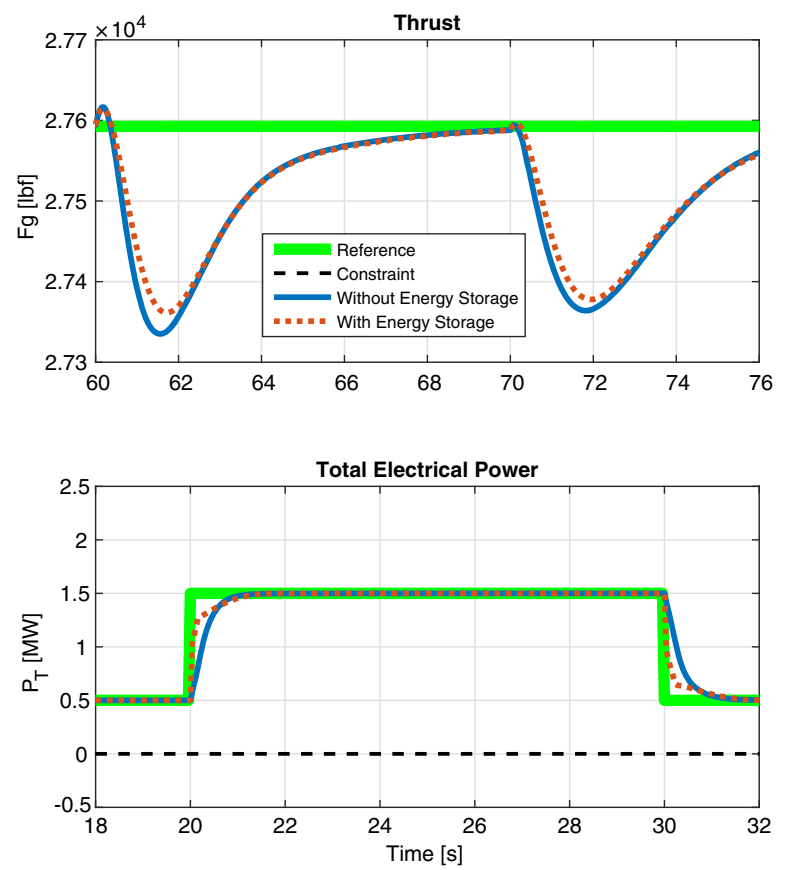

both types of energy storage elements. The simulation results are shown in Fig. 14.

As shown in the right subfigures in Fig. 14, with the energy storage element, the controller has better thrust and electrical power tracking. The comparison of the performance of all the cases is shown in Table 8. All energy storage element cases yield better performance than the case without the energy storage element, except for the second surge margin violation with the ultracapacitor pack. The second surge margin violation for the ultracapacitor pack is longer and larger than in the case without energy storage elements. This violation likely occurs because the ultracapacitor pack needs to be charged frequently, which requires the gas turbine engine to provide more output than in the case without the ultracapacitor. Thus, using the battery-ultracapacitor pack appears to be the preferred choice from the perspective of system response to thrust commands and electrical loads; and if the impact on weight, packaging, and cost is not considered.

Note that the energy storage elements are beneficial, based on our simulation results, despite the fact that their stored energy is limited in this study. Even more substantial benefits are expected for the energy storage elements with larger stored energy in future MEA.

Table 9 Parameters for LQR, MPC, offset MPC, and MMPC controllers

\begin{tabular}{lcccc}
\hline \hline & LQR & MPC & MMPC & Offset MPC \\
\hline Sampling time, s & 0.12 & 0.12 & 0.12 & 0.12 \\
Prediction horizon, steps & Inf & 30 & 30 & 30 \\
Constraint horizon, steps & N/A & 30 & 30 & 30 \\
Control horizon, steps & Inf & 10 & 10 & 10 \\
\hline \hline
\end{tabular}

Table 10 Performance comparison of single LQR, single MPC, MMPC, and single MPC with offset controllers

\begin{tabular}{lcccc}
\hline \hline & LQR & MPC & MMPC & Offset MPC \\
\hline$W_{f}, \mathrm{~kg}$ & 75.34 & 74.47 & 75.13 & 74.28 \\
$F g_{\text {AvgDev }}, \mathrm{lbf}$ & 170.06 & 719.72 & 497.93 & 718.93 \\
$P_{T_{\text {Avgev }}}, \mathrm{kW}$ & 7.50 & 101.14 & 92.18 & 101.14 \\
$n_{\text {smv }}$, times & 4 & 1 & 1 & 0 \\
$t_{d_{\text {suv }}}, \mathrm{s}$ & $1.44 / 1 / 5.4 / 0.48$ & 5.36 & 7.76 & 0 \\
SM $_{\text {Max } V}, \%$ & $3.9 / 3.86 / 1.24 / 0.97$ & 1.06 & 1.62 & 0 \\
\hline \hline
\end{tabular}



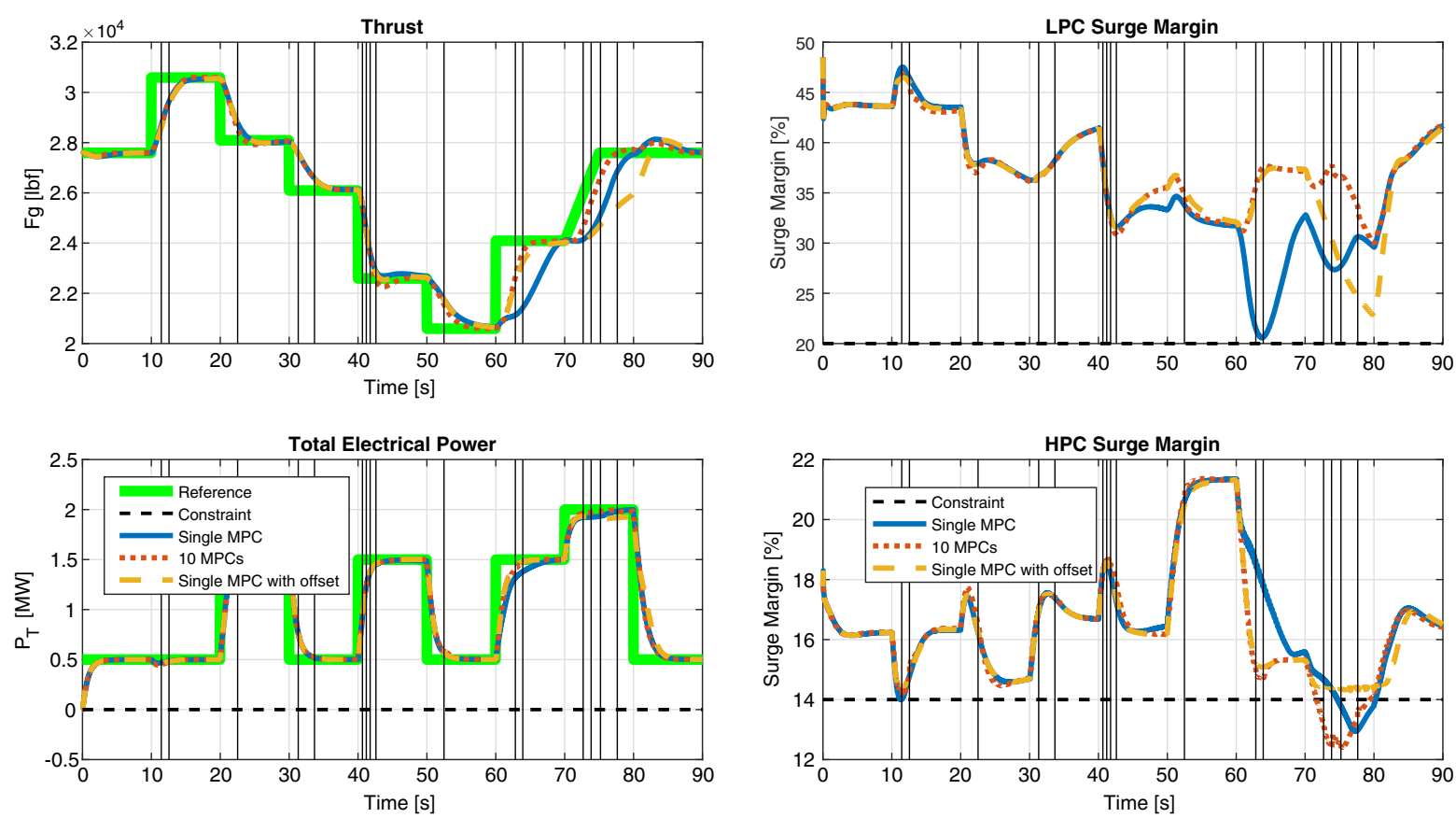

Fig. 15 Comparison of single MPC, MMPC, and single MPC with offset state controllers.

\section{Comparison of LQR, MPC, Offset MPC, and MMPC Controllers}

In this section, the LQR, MPC, offset MPC, and MMPC controllers are compared. The MMPC controller based on linearizations at multiple (10 in our case) operating points and the MPC controller with extra offset states are considered as potential approaches to better deal with the nonlinearities. For the purpose of quantifying the potential benefits of these design steps, the energy storage elements are not included in the analysis, and a broader range of thrust profiles is used compared to the previous simulations. The controller parameters are shown in Table 9 .

For all the controllers, the same parameters and constraints are used. The closed-loop performance comparison is shown in Table 10.

As expected, the LQR controller yields the best thrust and electrical load tracking, but it violates the surge margin constraints four times with a maximum of $3.9 \%$. It also consumes the largest fuel amount. The MPC controller is able to reduce the surge margin violations; nevertheless, it violates the surge margin constraint once at $5.36 \mathrm{~s}$ with a maximum violation of $1.06 \%$. This violation is likely due to the discrepancy between the prediction model and the actual nonlinear plant behavior. The MMPC design yields better thrust and electrical loads tracking than the MPC controller, but the surge margin constraint violation is longer and has larger magnitude than the MPC controller, which is likely due to surge margin prediction being insufficiently accurate. The offset MPC design yields very similar tracking performance to that of the MPC controller, and it has no violation of the surge margin constraint. The simulation results of the MPC, MMPC, and offset MPC controllers are shown in Fig. 15.

The black vertical lines indicate the switching time instants for MMPC controllers. As observed, switching does not cause improper behaviors of the system. For most of the simulation, all three controllers yield similar results, but the major differences can be found in the time interval between 60 and $80 \mathrm{~s}$. Specifically, between 60 and $70 \mathrm{~s}$, the MPC controller assumes that it does not have an available HPC surge margin because of inaccurate surge margin estimation due to being far from the operating point. Thus, it does not track the thrust setpoint aggressively, whereas the MMPC and offset MPC controllers are able to correctly account for the available HPC surge margin; hence, they track the thrust setpoint faster than the MPC controller. In the time interval between 70 and $80 \mathrm{~s}$, the MPC and MMPC controllers incorrectly assess that there is an available HPC surge margin, so they track the thrust setpoint aggressively; but, the offset MPC assesses that there is no available surge margin, so it tracks the thrust setpoint slowly to satisfy the HPC surge margin constraint and uses the available LPC surge margin. Thus, the offset MPC performs best in this simulation.

To confirm that the offset MPC is a good design choice, an energy storage element (the battery-ultracapacitor pack) is added to the offset MPC controller, and the cases without and with the energy storage element are compared to each other. The controller parameters and the constraints can be found in Tables $\underline{2}, \underline{7}$, and $\underline{9}$. The performance comparison is shown in Table 11 .

As shown in Table 11, both the thrust and the total electrical power tracking performance are improved with the addition of the energy storage, especially in terms of the total electrical power tracking performance. The fuel consumption is increased as a penalty for better tracking performance, but the increased amount is relatively small. The surge margin constraints are perfectly satisfied for both controllers. The simulation results of offset MPC controllers with and without energy storage elements are shown in Fig. 16.

As the left subfigures in Fig. 16 show, the offset $\overline{\mathrm{MPC}}$ with energy storage elements shows better thrust and total electrical power tracking. Both SOCs, especially for the ultracapacitor pack, violate the SOC constraint a small number of times to deal with transient thrust and electrical power changes, but they quickly recover to their constrained levels. As shown in the right subfigures, for both controllers, the power difference between the two generators stays within the optimal power split ranges for most of the time, which corresponds to safe and efficient operation.

\section{E. Simulation Results for Offset MPC with and Without Sensor Noise}

In this section, sensor noise is added to the measurements to verify the robustness of the offset MPC controller, and the responses with and without sensor noise are compared. Specifically, a zero mean

Table 11 Performance comparison of offset MPC with and without energy storage elements

\begin{tabular}{lcc}
\hline \hline & \multicolumn{2}{c}{ Offset MPC } \\
\cline { 2 - 3 } & Without & With BAT-UCAP pack \\
\hline$W_{f}, \mathrm{~kg}$ & 74.28 & 74.41 \\
$F g_{\text {AvgDev }}, \mathrm{lbf}$ & 718.93 & 672.91 \\
$P_{T_{\text {AvgDev }}, \mathrm{kW}}$ & 101.14 & 71.35 \\
$n_{\text {smv }}$, times & 0 & 0 \\
$t_{d_{\text {smy }},}, \mathrm{s}$ & 0 & 0 \\
$\mathrm{SM}_{\text {Max } V}, \%$ & 0 & 0 \\
\hline \hline
\end{tabular}



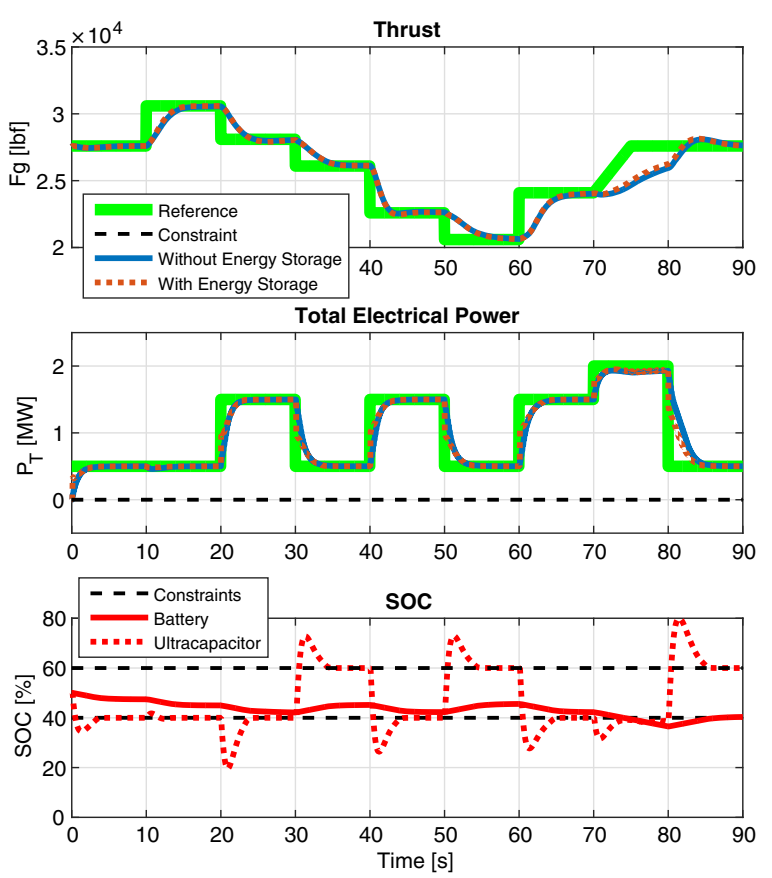

Fig. 16 Comparison of offset MPC with and without energy storage elements.
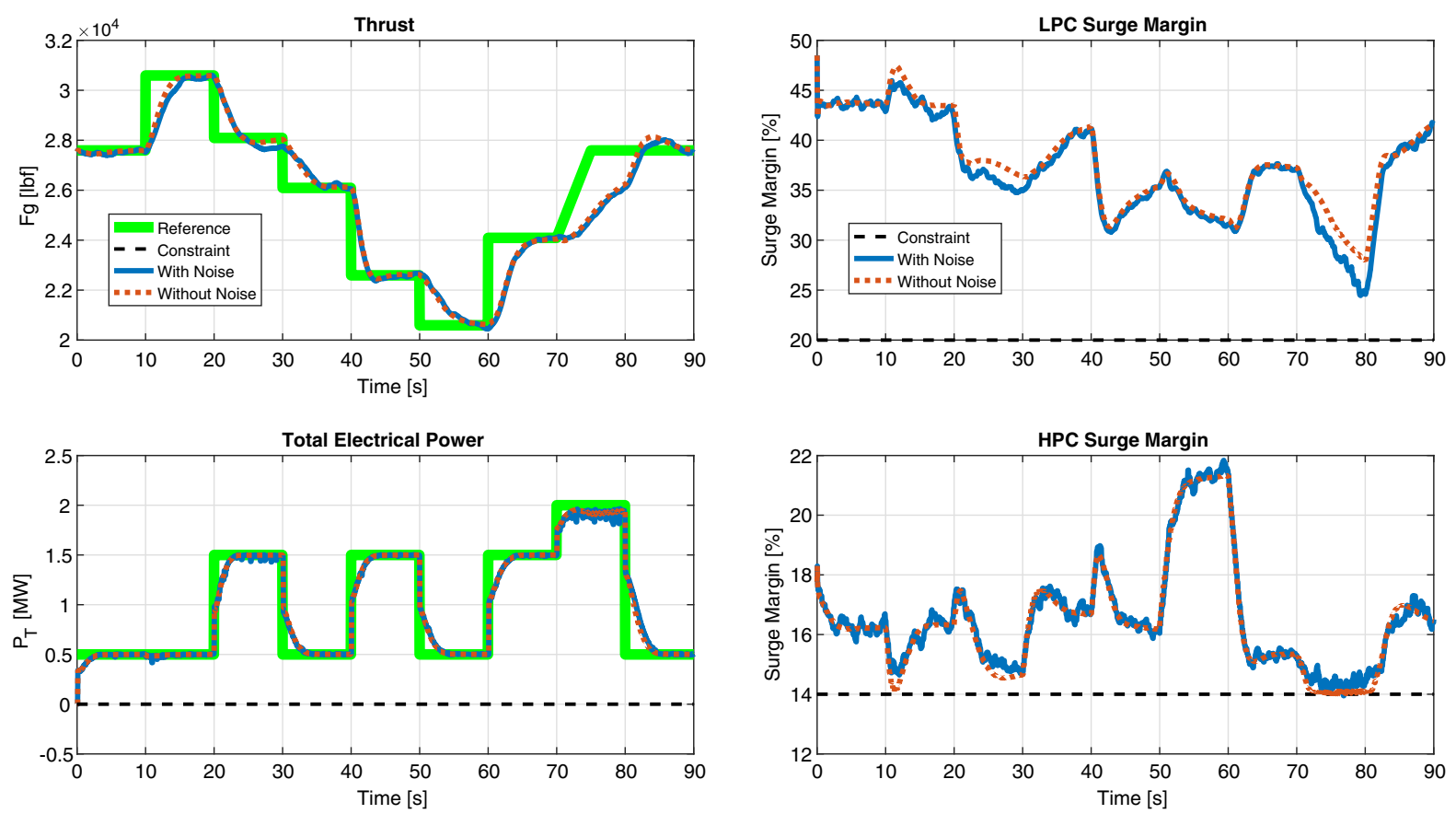

Fig. 17 Comparison of offset MPC controllers with and without sensor noise.

standard deviation Gaussian noise of $0.1 \%$ is added to the thrust, as well as the LPC and HPC surge margins measurements. The simulation results of offset MPC with and without sensor noise for the system configuration with the battery-ultracapacitor are shown in Fig. 17.

The simulation results show that the offset MPC controller is able to handle the sensor noise. In the time interval between 70 to $80 \mathrm{~s}$, when high electrical power is required and the thrust increment is requested near the HPC surge margin limit, some oscillations are observed because of the sensor noise, but the controller is able to handle the situation without having surge margin violations. Note that the computational delay can be accommodated by using the advanced-step MPC [40]; other delays, if they exist, can be handled by augmenting the discrete-time model with extra delay states.
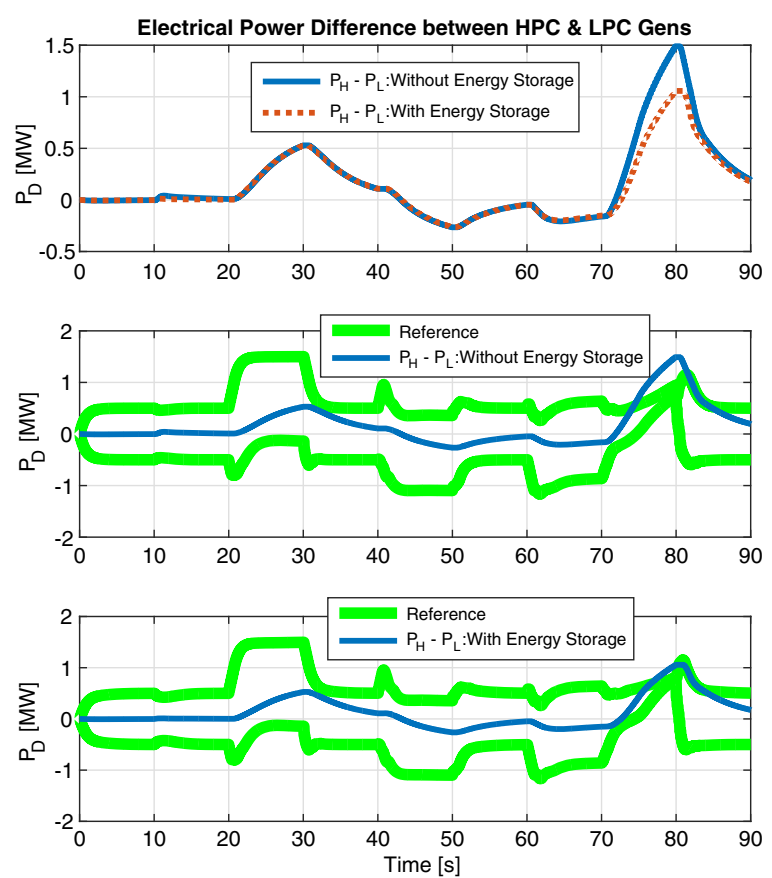
of tracking performance. The integrated LQR controller yielded better thrust and total electrical power tracking than the integrated MPC controller: however, with more surge margin constraint violations and without granting any protection against violation of other constraints.

To improve prediction model accuracy, MMPC and offset MPC design approaches were pursued. In the latter case, auxiliary offset states were used to represent the error between the linear model-based estimates of the constrained outputs and their actual value from the nonlinear model. The simulation results showed that a single MPC with offset states was able to satisfy the surge margin constraints, whereas MMPC, which is a more complex controller, had some constraint violations. Thus, the single rate-based offset MPC controller appeared to be the best strategy to control the system. The closed-loop system performance with different types of energy storage elements was also analyzed, with the combined battery and the ultracapacitor pack providing the best solution; however, the weight and size impact of such an approach need to be carefully analyzed. Including energy storage elements into the system improved performance. For instance, in the current simulations, the offset MPC controller with the battery-ultracapacitor pack improved the average thrust deviation by $6.4 \%$, the settling time of thrust by $3.47 \%$, the average total electrical power tracking by $29.45 \%$, and the settling time of the total electrical power by $8.65 \%$ as compared to an offset MPC controller applied to the system without energy storage and without any constraint violations. In addition, the offset MPC controller was able to handle sensor noise. The current results support the perspective that the aircraft architecture with dual generators attached to different gas turbine engine shafts and a batteryultracapacitor pack, controlled by a single offset MPC controller, is appealing in terms of fast, safe, and efficient thrust and electrical load delivery for future MEA and AEA.

\section{Acknowledgments}

The work was funded in part by The Boeing Company. The authors would like to thank Brandon Hencey from the U.S. Air Force Research Laboratory for technical discussions and comments.

\section{References}

[1] Moir, I., and Seabridge, A., Aircraft Systems: Mechanical, Electrical and Avionics Subsystems Integration, 3rd ed., Wiley, New York, 2008, Chaps. 1, 5, 10.

[2] Rosero, J. A., Ortega, J. A., Aldabas, E., and Romeral, L., "Moving Towards a More Electric Aircraft," IEEE Aerospace and Electronic Systems Magazine, Vol. 22, No. 3, March 2007, pp. 3-9. doi:10.1109/MAES.2007.340500

[3] Luongo, C. A., Masson, P. J., Nam, T., Mavris, D., Kim, H. D., Brown, G. V., Waters, M., and Hall, D., "Next Generation MoreElectric Aircraft: A Potential Application for HTS Superconductors," IEEE Transactions on Applied Superconductivity, Vol. 19, No. 3, June 2009, pp. 1055-1068. doi:10.1109/TASC.2009.2019021

[4] Felder, J., Kim, H., and Brown, G., "Turboelectric Distributed Propulsion Engine Cycle Analysis for Hybrid-Wing-Body Aircraft," AIAA Aerospace Sciences Meeting Including the New Horizons Forum and Aerospace Exposition, AIAA Paper 2009-1132, Jan. 2009. doi:10.2514/6.2009-1132

[5] Kim, H. D., "Distributed Propulsion Vehicles," 27th International Congress of the Aeronautical Sciences, ICAS, Bonn, Germany, Sept. 2010.

[6] McNab, I. R., "Pulsed Power for Electric Guns," IEEE Transactions on Magnetics, Vol. 33, No. 1, Jan. 1997, pp. 453-460. doi:10.1109/20.560055

[7] "Technology Horizons: A Vision for Air Force Science and Technology 2010-30," U.S. Air Force TR AF/ST-TR-10-01-PR, 2010.

[8] Behbahani, A., et al., "Status, Vision, and Challenges of an Intelligent Distributed Engine Control Architecture," Soc. of Automotive Engineers TP 2007-01-3859, Warrendale, PA, 2007. doi:10.4271/2007-01-3859

[9] Behbahani, A. R., Von Moll, A., Zeller, R., and Ordo, J., "Aircraft Integration Challenges and Opportunities for Distributed Intelligent Control, Power, Thermal Management, Diagnostic and Prognostic
Systems," Soc. of Automotive Engineers TP 2014-01-2161, Warrendale, PA, 2014.

doi:10.4271/2014-01-2161

[10] Litt, J. S., Simon, D. L., Garg, S., Guo, T.-H., Mercer, C., Millar, R., Behbahani, A., Bajwa, A., and Jensen, D. T., "A Survey of Intelligent Control and Health Management Technologies for Aircraft Propulsion Systems," Journal of Aerospace Computing, Information, and Communication, Vol. 1, No. 12, 2004, pp. 543-563. doi:10.2514/1.13048

[11] DeSimio, M. P., Hencey, B. M., and Parry, A. C., "Online Prognostics for Fuel Thermal Management System," ASME 2015 Dynamic Systems and Control Conference, American Soc. of Mechanical Engineers Paper V001T08A003, Fairfield, NJ, Oct. 2015. doi:10.1115/DSCC2015-9842

[12] Richter, H., Advanced Control of Turbofan Engines, 1st ed., Springer, New York, 2012, Chap. 9.

[13] DeCastro, J. A., "Rate-Based Model Predictive Control of Turbofan Engine Clearance," Journal of Propulsion and Power, Vol. 23, No. 4, 2007, pp. 804-813. doi: $10.2514 / 1.25846$

[14] Fuller, J., Das, I., Potra, F., and Ji, J., "System and Method of Applying Interior Point Method for Online Model Predictive Control of Gas Turbine Engines," U.S. Patent Application 11/150,703, June 2005.

[15] Huang, M., Zaseck, K., Butts, K., and Kolmanovsky, I., "Rate-Based Model Predictive Controller for Diesel Engine Air Path: Design and Experimental Evaluation," IEEE Transactions on Control Systems Technology, Vol. 24, No. 6, March 2016, pp. 1922-1935. doi:10.1109/TCST.2016.2529503

[16] Herceg, M., Kvasnica, M., Jones, C. N., and Morari, M., "MultiParametric Toolbox (MPT) 3.0," European Control Conference, IEEE Publ., Piscataway, NJ, July 2013, pp. 502-510, http://control.ee.ethz.ch/ $\sim$ mpt.

[17] Mitcham, A. J., and Cullen, J. J. A., "Permanent Magnet Generator Options for the More Electric Aircraft," International Conference on Power Electronics, Machines and Drives, IET, Stevenage, U.K., June 2002, pp. 241-254. doi:10.1049/cp:20020121

[18] Rakhra, P., Norman, P. J., Galloway, S. J., and Burt, G. M., "Modelling and Simulation of a MEA Twin Generator UAV Electrical Power System," International Proceedings of Universities' Power Engineering Conference, VDE, Berlin, Germany, Sept. 2011, pp. 1-5.

[19] Muehlbauer, K., and Gerling, D., "Two-Generator-Concepts for Electric Power Generation in More Electric Aircraft Engine," International Conference on Electrical Machines, IEEE Publ., Piscataway, NJ, Sept. 2010, pp. 1-5. doi:10.1109/ICELMACH.2010.5607966

[20] Alnajjar, M., and Gerling, D., "Control of Three-Source High Voltage Power Network for More Electric Aircraft," International Symposium on Power Electronics, Electrical Drives, Automation and Motion, IEEE Publ., Piscataway, NJ, June 2014, pp. 232-237. doi:10.1109/SPEEDAM.2014.6871906

[21] Kim, J. S., Powell, K. M., and Edgar, T. F., "Nonlinear Model Predictive Control for a Heavy-Duty Gas Turbine Power Plant," American Control Conference, IEEE Publ., Piscataway, NJ, June 2013, pp. 2952-2957. doi:10.1109/ACC.2013.6580283

[22] Thirunavukarasu, E., Fang, R., Khan, J. A., and Dougal, R., "Evaluation of Gas Turbine Engine Dynamic Interaction with Electrical and Thermal System," Electric Ship Technologies Symposium, IEEE Publ., Piscataway, NJ, April 2013, pp. 442-448. doi:10.1109/ESTS.2013.6523773

[23] Norman, P. J., Galloway, S. J., Burt, G. M., Hill, J. E., and Trainer, D. R., "Evaluation of the Dynamic Interactions Between Aircraft Gas Turbine Engine and Electrical System," IET Conference on Power Electronics, Machines and Drives, IET, Stevenage, U.K., April 2008, pp. 671-675. doi: $10.1049 / \mathrm{cp}: 20080606$

[24] Todd, R., Wu, D., dos Santos Girio, J. A., Poucand, M., and Forsyth, A. J., "Supercapacitor-Based Energy Management for Future Aircraft Systems," Applied Power Electronics Conference and Exposition, IEEE Publ., Piscataway, NJ, Feb. 2010, pp. 1306-1312. doi:10.1109/APEC.2010.5433398

[25] Shahsavari, B., Maasoumy, M., Sangiovanni-Vincentelli, A., and Horowitz, R., "Stochastic Model Predictive Control Design for Load Management System of Aircraft Electrical Power Distribution," American Control Conference, IEEE Publ., Piscataway, NJ, July 2015, pp. 3649-3655. doi:10.1109/ACC.2015.7171897 
[26] Jaw, L. C., and Mattingly, J., Aircraft Engine Controls: Design, System Analysis, and Health Monitoring, 1st ed., AIAA, Reston, VA, 2010, Chaps. 2-7.

[27] Chapman, J. W., Lavelle, T. M., Litt, J. S., May, R. D., and Guo, T.-H., "Propulsion System Simulation Using the Toolbox for the Modeling and Analysis of Thermodynamic Systems (T-MATS)," Propulsion and Energy Forum, AIAA Paper 2014-3929, July 2014. doi:10.2514/6.2014-3929

[28] Zinnecker, A., Chapman, J. W., Lavelle, T. M., and Litt, J. S., "Development of a Twin-Spool Turbofan Engine Simulation Using the Toolbox for Modeling and Analysis of Thermodynamic Systems (T-MATS)," Propulsion and Energy Forum, AIAA Paper 2014-3930, July 2014. doi:10.2514/6.2014-3930

[29] Chapman, J. W., Lavelle, T. M., Litt, J. S., and Guo, T.-H., "A Process for the Creation of T-MATS Propulsion System Models from NPSS Data," Propulsion and Energy Forum, AIAA Paper 2014-3931, July 2014. doi:10.2514/6.2014-3931

[30] Seok, J., Kolmanovsky, I., and Girard, A., "Integrated/Coordinated Control of Aircraft Gas Turbine Engine and Electrical Power System: Towards Large Electrical Load Handling," IEEE Conference on Decision and Control, IEEE Publ., Piscataway, NJ, Dec. 2016, pp. 3183-3189. doi:10.1109/CDC.2016.7798747

[31] Corbett, M., Lamm, P., McNichols, J., Boyd, M., and Wolff, M., "Effects of Transient Power Extraction on an Integrated Hardware-in-the-Loop Aircraft/Propulsion/Power System," Soc. of Automotive Engineers TP 2008-01-2926, Warrendale, PA, 2008. doi:10.4271/2008-01-2926

[32] Vahidi, A., Kolmanovsky, I., and Stefanopoulou, A., "Constraint Handling in a Fuel Cell System: A Fast Reference Governor Approach," IEEE Transactions on Control Systems Technology, Vol. 15, No. 1, 2007, pp. 86-98. doi:10.1109/TCST.2006.883242

[33] Garone, E., Di Cairano, S., and Kolmanovsky, I., "Reference and Command Governors for Systems with Constraints: A Survey of
Their Theory and Application," Automatica, Vol. 75, No. 1, 2017, pp. 306-328.

doi:10.1016/i.automatica.2016.08.013

[34] DeLaat, J. C., Southwick, R. D., and Gallops, G. W., "High Stability Engine Control (HISTEC)," 32nd Joint Propulsion Conference Cosponsored by AIAA, ASME and SAE, AIAA Paper 1996-2586, July 1996. doi:10.2514/6.1996-2586

[35] Ortner, P., and del Re, L., "Predictive Control of a Diesel Engine Air Path," IEEE Transactions on Control Systems Technology, Vol. 15, No. 3, 2007, pp. 449-456. doi: $10.1109 /$ TCST.2007.894638

[36] Di Cairano, S., and Tseng, H., "Driver-Assist Steering by Active Front Steering and Differential Braking: Design, Implementation and Experimental Evaluation of a Switched Model Predictive Control Approach," IEEE Conference on Decision and Control, IEEE Publ., Piscataway, NJ, Dec. 2010, pp. 2886-2891 doi:10.1109/CDC.2010.5716954

[37] Huang, M., Nakada, H., Polavarapu, S., Choroszucha, R., Butts, K., and Kolmanovsky, I., "Towards Combining Nonlinear and Predictive Control of Diesel Engines," American Control Conference, IEEE Publ., Piscataway, NJ, June 2013, pp. 2846-2853. doi:10.1109/ACC.2013.6580266

[38] "Nanophosphate Lithium Ion Prismatic Pouch Cell AMP20M1HD-A," http://liionbms.com/pdf/a123/AMP20M1HD-A.pdf [retrieved 20 April 2017].

[39] "Datasheet: K2 Ultracapacutors-2.85V/3400F," Maxwell Technologies Document 3000619-EN.3, San Diego, CA, http://www.maxwell.com/ images/documents/K2 2 85V DS 3000619EN 3 .pdf [retrieved 20 April 2017].

[40] Zavala, V. M., and Biegler, L. T., "The Advanced-Step NMPC Controller: Optimality, Stability and Robustness," Automatica, Vol. 45, No. 1, 2009, pp. 86-93. doi:10.1016/j.automatica.2008.06.011 